\title{
MATHEMATICAL ANALYSIS AND TREATMENT FOR THE TRUE AND SPURIOUS EIGENEQUATIONS OF CIRCULAR PLATES BY THE MESHLESS METHOD USING RADIAL BASIS FUNCTION
}

\author{
Jeng-Tzong Chen*, Ying-Te Lee, I-Lin Chen, and Kue-Hong Chen
}

\begin{abstract}
In this paper, a meshless method for solving the eigenproblems of plate vibration using the radial basis function (RBF) is proposed. By employing the RBF in the imaginary-part fundamental solution, spurious eigenequations in conjunction with the true ones are obtained at the same time. Mathematical analysis for the appearance of spurious eigenequations by using degenerate kernel and circulant is done through a circular plate for a discrete system. In order to obtain the true and spurious eigenequations, six $\left(C_{2}^{4}\right)$ formulations (either two combinations from the four types of potentials, single, double, triple and quadruple) of meshless methods are employed in conjunction with the SVD technique. The spurious eigenequation in each formulation is found and is filtered out by using the SVD updating technique. Three cases, clamped, simply-supported and free circular plates, are demonstrated to check the validity of the meshless methods.
\end{abstract}

Key Words: meshless method, radial basis function, spurious eigenequation, plate vibration, SVD technique.

\section{INTRODUCTION}

Mesh generation of a complicated geometry is always time consuming in the stage of model creation for engineers in treating engineering problems by utilizing numerical methods, e.g., the finite difference method (FDM), finite element method (FEM) and boundary element method (BEM). In the last decade, researchers have paid attention to the meshless method to reduce the effort in model creation. The initial idea of a meshless method dates back to the smooth particle hydrodynamics (SPH) method for modeling astrophysical phenomena (Gingold and Maraghan, 1977). Basically, the meshless method is

*Corresponding author. (Tel: 886-2-24622192-6177; Fax: 8862-24632375; Email: jtchen@mail.ntou.edu.tw)

J. T. Chen and Y. T. Lee are with the Department of Harbor and River Engineering, National Taiwan Ocean University, Keelung, Taiwan 202, R.O.C.

I. L. Chen is with the Department of Naval Architecture, National Kaohsiung Marine University, Kaohsiung, Taiwan 814 , R.O.C.

K. H. Chen is with the Hydrotech Research Institute, National Taiwan University, Taipei, Taiwan 106, R.O.C. classified among the domain-based methods, including the element-free Galerkin method (Belystcho et al., 1994), the reproducing kernel method (Liu et al., 1995), and boundary-based methods including the boundary node method (Mukherjee and Mukherjee, 1997), the meshless local Petrov-Galerkin approach (Atluri and Zhu, 1998), the local boundary integral equation method (Sladek et al., 2000), the RBF approach (Chen, 2000b; Chen and Tanaka, 2000a; 2000b; Golberg et al., 2000), and the boundary knot method (BKM) (Chen, 2000c; Hon and Chen, 2003).

Recently, the method of fundamental solutions (MFS), another numerical technique has become as popular as FDM, FEM and BEM. It is well known that the method of fundamental solutions can deal with engineering problems when a fundamental solution is known. This method was attributed to Kupradze in 1964. The method of fundamental solutions can be applied to potential (Fairweather and Karageorghis, 1998), Helmholtz (Karageorghis, 2001), diffusion (Chen et al., 1998), biharmonic (Poullikkas et al., 1998) and elasticity problems (Kupradze, 1964). The method of fundamental solutions can be seen as one kind of meshless method. The basic idea is to approximate 
the solution by a linear superposition of fundamental solution with source located outside the domain of the problem. Moreover, it has some advantages over boundary element method, e.g., no singularity, no boundary integrals and mesh-free model.

Many researchers have tried, over a long period of time, to develop efficient methods for calculating the eigenvalues of plate vibrations. Readers can consult a review article by Leissa (1969). The exact form for the eigenequation is not available except in very limited cases. Numerical methods are fallen back on to solve the real problem. A large number of investigators have studied the problem numerically, e.g., Beskos (1991), Hutchinson (1988), Chen et al. (2004) and Lee et al. (2002). Until recent years, the meshless method was adopted to solve the plate eigenproblem. Kang and Lee (2001) used the so-called NDIF method to deal with the plate problem. Later, Chen et al. (2003) found that the NDIF method is equivalent to the imaginarypart kernel method. Almost at the same time, Chen (2000) adopted the imaginary-part kernel to develop the so-called boundary knot method (BKM). Chen et al. (2002) pointed out that either real-part or imaginary-part kernel methods result in spurious solutions. The spurious eigenequation for the membrane and acoustics in the real-part and imaginary-part kernel method has been studied in detail by Chen's group and circumvented by many regularization techniques, e.g., dual method (Chen, 2000a), domain decomposition (Chang et al., 1999), SVD updating technique (Chen et al., 1999) and CHEEF method (Chen et al., 2001). Kang's group (Kang and Lee, 2000a; 2000b; 2001) also has developed remedies to overcome the problem of spurious solutions. However, many techniques have not been applied to deal with the plate eigenproblems.

In this paper, the appearance of spurious eigenequations is mathematically derived through a circular plate. The imaginary-part fundamental solution is chosen as a radial basis function to deal with the plate vibration. Six versions of meshless method arrived at by adopting two from the four types of potentials (single, double, triple and quadruple) are proposed in conjunction with the SVD updating technique. The spurious eigenequation embedded in the six formulations will be derived. To filter out the spurious eigenequation, SVD updating will be adopted for the three cases, clamped, simply-supported and free plates. The obtained true eigenequation will be compared with those of the exact solution in Leissa's book (Leissa, 1969).

\section{MESHLESS METHOD USING RADIAL BASIS FUNCTION OF THE IMAGINARY-PART FUNDAMENTAL SOLUTION}

The governing equation for a free flexural vibration of a uniform thin plate is written as follows:

$$
\nabla^{4} u(x)=\lambda^{4} u(x), x \in \Omega
$$

where $u$ is the lateral displacement, $\lambda^{4}=\frac{\omega^{2} \rho_{0} h}{D}, \lambda$ is the frequency parameter, $\omega$ is the circular frequency, $\rho_{0}$ is the surface density, $D$ is the flexural rigidity expressed as $D=\frac{E h^{3}}{12\left(1-v^{2}\right)}$ in terms of Young's modulus $E$, the Poisson ratio $v$ and the plate thickness $h$, and $\Omega$ is the domain of the thin plate.

Based on the indirect formulation, the displacement field of plate vibration can be represented by

$$
u(x)=\sum_{j=1}^{2 N} P\left(s_{j}, x\right) \phi_{j}+\sum_{j=1}^{2 N} Q\left(s_{j}, x\right) \psi_{j}
$$

where $2 N$ is the number of boundary nodes, $s$ and $x$ are the source and field points, respectively. $\phi_{j}$ and $\psi_{j}$ are the unknown densities with respect to $P$ and $Q$. The two kernels ( $P$ and $Q$ ) are obtained from combinations of the following four kernels,

$$
\begin{aligned}
& U(s, x)=\operatorname{Im}\left[\frac{i}{8 \lambda^{2}}\left(H_{0}^{(1)}(\lambda r)+H_{0}^{(2)}(i \lambda r)\right)\right] \\
& \Theta(s, x)=K_{\theta, s}(U(s, x)) \\
& M(s, x)=K_{m, s}(U(s, x)) \\
& V(s, x)=K_{v, s}(U(s, x))
\end{aligned}
$$

in which the components of fundamental solution (Kitahara, 1985), $H_{0}^{(1)}$ and $H_{0}^{(2)}$ are the first kind and second kind zeroth-order Hankel functions, respectively, Im denotes the imaginary part, and $K_{\theta}(\cdot), K_{m}(\cdot)$ and $K_{v}(\cdot)$ mean the operators which are defined as follows:

$$
\begin{aligned}
& K_{\theta}(\cdot)=\frac{\partial(\cdot)}{\partial n} \\
& K_{m}(\cdot)=v \nabla^{2}(\cdot)+(1-v) \frac{\partial^{2}(\cdot)}{\partial n^{2}} \\
& K_{v}(\cdot)=\frac{\partial \nabla^{2}(\cdot)}{\partial n}+(1-v) \frac{\partial}{\partial t}\left(\frac{\partial^{2}(\cdot)}{\partial n \partial t}\right)
\end{aligned}
$$

where $n$ and $t$ are the normal vector and tangential vector, respectively. The operators $K_{\theta}(\cdot), K_{m}(\cdot)$ and $K_{v}(\cdot)$ can be applied to $U, \Theta, M$ and $V$ kernels to generate sixteen kernels in total as shown in Fig. 1. By applying the three operators in Eqs. (7), (8) and (9) to Eq. (2), we have

$$
\begin{aligned}
& \theta(x)=K_{\theta}(u(x)) \\
& m(x)=K_{m}(u(x))
\end{aligned}
$$




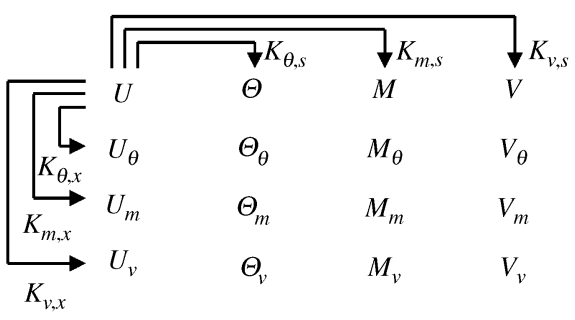

Fig. 1 The construction of the sixteen kernels

$$
v(x)=K_{v}(u(x))
$$

where $\theta, m$ and $v$ denote the slope, normal moment and effective shear force. After collocating the point $x$ on the boundary, Eq. (2) and Eqs. (10)-(12) can be rewritten in matrix forms of:

$$
\begin{aligned}
& \{u\}=[P]\{\phi\}+[Q]\{\psi\} \\
& \{\theta\}=\left[P_{\theta}\right]\{\phi\}+\left[Q_{\theta}\right]\{\psi\} \\
& \{m\}=\left[P_{m}\right]\{\phi\}+\left[Q_{m}\right]\{\psi\} \\
& \{v\}=\left[P_{v}\right]\{\phi\}+\left[Q_{v}\right]\{\psi\}
\end{aligned}
$$

where $\{\phi\}$ and $\{\psi\}$ are the vectors of undetermined coefficients, the subscripts $\theta, m$ and $v$ of $P$ and $Q$ denote the operators, $K_{\theta}, K_{m}$ and $K_{v}$, respectively. By matching the boundary conditions of the clamped plate ( $u=0$ and $\theta=0$ ) with a radius $a$ and choosing the $U$ and $\Theta$ for the $P$ and $Q$ kernels, we have

$$
\{0\}=[U]\{\phi\}+[\Theta]\{\psi\}
$$

$$
\{0\}=\left[U_{\theta}\right]\{\phi\}+\left[\Theta_{\theta}\right]\{\psi\}
$$

By assembling Eqs. (17) and (18) together, we have

$$
\left[S M_{1}^{c}\right]\left\{\begin{array}{l}
\phi \\
\psi
\end{array}\right\}=\{0\}
$$

where the superscript " $c$ " denotes the clamped case and

$$
\left[S M_{1}^{c}\right]=\left[\begin{array}{cc}
U & \Theta \\
U_{\theta} & \Theta_{\theta}
\end{array}\right]_{4 N \times 4 N}
$$

the determinant of the matrix versus eigenvalue must be zero to obtain the nontrivial solution, i.e.,

$$
\operatorname{det}\left[S M_{1}^{c}\right]=0
$$

By plotting the determinant versus the frequency parameter, the curve drops at the positions of eigenvalues. Since the $P$ and $Q$ kernels can be selected from any two of the four types of kernels ( $U$, $\Theta, M$ and $V)$, six $\left(C_{2}^{4}\right)$ formulations can be considered. Similarly, the eigenvalues of simply-supported and free plates can be solved by using the six formulations.

\section{ANALYTICAL DERIVATIONS FOR THE EIGENEQUATION OF A CIRCULAR PLATE}

For the circular plate, we can express $x=(\rho, \theta)$ and $s=(\bar{\rho}, \bar{\theta})$ in terms of polar coordinates. The $U$ kernel function can be expressed in terms of the degenerate kernels as shown below (Chen et al., 2002):

$$
\begin{gathered}
U(s, x)=\left\{\begin{array}{l}
U^{I}(\bar{\rho}, \bar{\theta} ; \rho, \theta)=\frac{1}{8 \lambda^{2}} \sum_{\ell=-\infty}^{\infty}\left[J_{\ell}(\lambda \bar{\rho}) J_{\ell}(\lambda \rho)+(-1)^{\ell} I_{\ell}(\lambda \bar{\rho}) I_{\ell}(\lambda \rho)\right] \cos (\ell(\theta-\bar{\theta})), \quad \bar{\rho}>\rho \\
U^{E}(\bar{\rho}, \bar{\theta} ; \rho, \theta)=\frac{1}{8 \lambda^{2}} \sum_{\ell=-\infty}^{\infty}\left[J_{\ell}(\lambda \rho) J_{\ell}(\lambda \bar{\rho})+(-1)^{\ell} I_{\ell}(\lambda \rho) I_{\ell}(\lambda \bar{\rho})\right] \cos (\ell(\theta-\bar{\theta})), \quad \bar{\rho}<\rho
\end{array}\right. \\
\Theta(s, x)=\left\{\begin{array}{l}
\Theta^{I}(\bar{\rho}, \bar{\theta} ; \rho, \theta)=\frac{1}{8 \lambda^{2}} \sum_{\ell=-\infty}^{\infty}\left[J_{\ell}^{\prime}(\lambda \bar{\rho}) J_{\ell}(\lambda \rho)+(-1)^{\ell} I_{\ell}(\lambda \rho) I_{\ell}^{\prime}(\lambda \bar{\rho})\right] \cos (\ell(\theta-\bar{\theta})), \quad \rho>\rho \\
\Theta^{E}(\bar{\rho}, \bar{\theta} ; \rho, \theta)=\frac{1}{8 \lambda^{2}} \sum_{\ell=-\infty}^{\infty}\left[J_{\ell}(\lambda \rho) J_{\ell}^{\prime}(\lambda \bar{\rho})+(-1)^{\ell} I_{\ell}^{\prime}(\lambda \bar{\rho}) I_{\ell}(\lambda \rho)\right] \cos (\ell(\theta-\bar{\theta})), \quad \rho<\rho
\end{array}\right.
\end{gathered}
$$


where $J_{\ell}$ and $I_{\ell}$ denote the $\ell$ th order first kind Bessel and modified Bessel functions, the superscripts " $I$ " and " $E$ " denote the interior $(\bar{\rho}>\rho)$ and exterior domains $(\bar{\rho}<\rho)$, respectively.

Since the rotation symmetry is preserved for a circular boundary, the sixteen influence matrices are denoted by $[U],[\Theta],[M],[V],\left[U_{\theta}\right],\left[\Theta_{\theta}\right],\left[M_{\theta}\right],\left[V_{\theta}\right]$, $\left[U_{m}\right],\left[\Theta_{m}\right],\left[M_{m}\right],\left[V_{m}\right],\left[U_{v}\right],\left[\Theta_{v}\right],\left[M_{v}\right]$ and $\left[V_{v}\right]$ of the circulants with the elements

$$
K_{i j}=K\left(\bar{\rho}, \bar{\theta}_{j} ; \rho, \theta_{i}\right)
$$

where the kernel $K$ can be any of the sixteen kernels, $U, \Theta, M, V, U_{\theta}, \Theta_{\theta}, M_{\theta}, V_{\theta}, U_{m}, \Theta_{m}, M_{m}, V_{m}, U_{v}, \Theta_{v}$, $M_{v}$ and $V_{v}$, as shown in Fig. $1, \theta_{i}$ and $\bar{\theta}_{j}$ are the angles of observation and boundary points, respectively. By superimposing $2 N$ lumped strength along the boundary, we have the influence matrix,

$$
[K]=\left[\begin{array}{cccccc}
a_{0} & a_{1} & a_{2} & \cdots & a_{2 N-2} & a_{2 N-1} \\
a_{2 N-1} & a_{0} & a_{1} & \cdots & a_{2 N-3} & a_{2 N-2} \\
a_{2 N-2} & a_{2 N-1} & a_{0} & \cdots & a_{2 N-4} & a_{2 N-3} \\
\vdots & \vdots & \vdots & \ddots & \vdots & \vdots \\
a_{1} & a_{2} & a_{3} & \cdots & a_{2 N-1} & a_{0}
\end{array}\right]
$$

where the elements of the first row can be obtained by

$$
a_{j-i}=K\left(s_{j}, x_{i}\right)
$$

The matrix $[K]$ in Eq. (25) is found to be a symmetric circulant (Kuo et al., 2000) since the rotational symmetry for the influence coefficients is considered. By using degenerate kernels and the orthogonal property, the eigenvalues of the matrices, $[U],[\Theta],[M],[V]$, $\left[U_{\theta}\right],\left[\Theta_{\theta}\right],\left[M_{\theta}\right],\left[V_{\theta}\right],\left[U_{m}\right],\left[\Theta_{m}\right],\left[M_{m}\right],\left[V_{m}\right],\left[U_{v}\right]$, $\left[\Theta_{v}\right],\left[M_{v}\right]$ and $\left[V_{v}\right]$, can be, respectively, obtained as follows:

$$
\begin{aligned}
\lambda_{\ell}^{[U]}= & \frac{N}{4 \lambda^{2}}\left[J_{\ell}(\lambda a) J_{\ell}(\lambda a)+(-1)^{\ell} I_{\ell}(\lambda a) I_{\ell}(\lambda a)\right] \\
\lambda_{\ell}^{[\Theta]}= & \frac{N}{4 \lambda}\left[J_{\ell}(\lambda a) J_{\ell}^{\prime}(\lambda a)+(-1)^{\ell} I_{\ell}(\lambda a) I_{\ell}^{\prime}(\lambda a)\right] \\
\lambda_{\ell}^{[M]}= & \frac{N}{4}\left\{\left[J_{\ell}(\lambda a) J_{\ell}^{\prime \prime}(\lambda a)+(-1)^{\ell} I_{\ell}(\lambda a) I_{\ell}^{\prime \prime}(\lambda a)\right]\right. \\
& +\frac{v}{\lambda a}\left[J_{\ell}(\lambda a) J_{\ell}^{\prime}(\lambda a)+(-1)^{\ell} I_{\ell}(\lambda a) I_{\ell}^{\prime}(\lambda a)\right] \\
& \left.-\frac{\ell^{2} v}{\lambda^{2} a^{2}}\left[J_{\ell}(\lambda a) J_{\ell}(\lambda a)+(-1)^{\ell} I_{\ell}(\lambda a) I_{\ell}(\lambda a)\right]\right\}
\end{aligned}
$$

$$
\begin{aligned}
& \lambda_{\ell}^{[V]} \\
& =\frac{N}{4}\left\{\lambda\left[J_{\ell}(\lambda a) J_{\ell}^{\prime \prime \prime}(\lambda a)+(-1)^{\ell} I_{\ell}(\lambda a) I_{\ell}^{\prime \prime \prime}(\lambda a)\right]\right. \\
& +\frac{1}{a}\left[J_{\ell}(\lambda a) J_{\ell}^{\prime \prime}(\lambda a)+(-1)^{\ell} I_{\ell}(\lambda a) I_{\ell}^{\prime \prime}(\lambda a)\right] \\
& -\frac{1}{\lambda a^{2}}\left[J_{\ell}(\lambda a) J_{\ell}^{\prime}(\lambda a)+(-1)^{\ell} I_{\ell}(\lambda a) I_{\ell}^{\prime}(\lambda a)\right] \\
& +\frac{\ell^{2}(3-v)}{\lambda^{2} a^{3}}\left[J_{\ell}(\lambda a) J_{\ell}(\lambda a)+(-1)^{\ell} I_{\ell}(\lambda a) I_{\ell}(\lambda a)\right] \\
& \left.-\frac{\ell^{2}(2-v)}{\lambda a^{2}}\left[J_{\ell}(\lambda a) J_{\ell}^{\prime}(\lambda a)+(-1)^{\ell} I_{\ell}(\lambda a) I_{\ell}^{\prime}(\lambda a)\right]\right\} \\
& \mu_{\ell}^{[U]}=\frac{N}{4 \lambda}\left[J_{\ell}^{\prime}(\lambda a) J_{\ell}(\lambda a)+(-1)^{\ell} I_{\ell}^{\prime}(\lambda a) I_{\ell}(\lambda a)\right] \\
& \mu_{\ell}^{[\Theta]}=\frac{N_{4}}{4}\left[J_{\ell}^{\prime}(\lambda a) J_{\ell}^{\prime}(\lambda a)+(-1)^{\ell} I_{\ell}^{\prime}(\lambda a) I_{\ell}^{\prime}(\lambda a)\right] \\
& \mu_{\ell}^{[M]}=\frac{N}{4}\left\{\lambda\left[J_{\ell}^{\prime}(\lambda a) J_{\ell}^{\prime \prime}(\lambda a)+(-1)^{\ell} I_{\ell}^{\prime}(\lambda a) I_{\ell}^{\prime \prime}(\lambda a)\right]\right. \\
& +\frac{v}{a}\left[J_{\ell}^{\prime}(\lambda a) J_{\ell}^{\prime}(\lambda a)+(-1)^{\ell} I_{\ell}^{\prime}(\lambda a) I_{\ell}^{\prime}(\lambda a)\right] \\
& \left.-\frac{\ell^{2} v^{2}}{\lambda a^{2}}\left[J_{\ell}^{\prime}(\lambda a) J_{\ell}(\lambda a)+(-1)^{\ell} I_{\ell}^{\prime}(\lambda a) I_{\ell}(\lambda a)\right]\right\}
\end{aligned}
$$

$$
\begin{aligned}
\mu_{\ell}^{[V]} & =\frac{N}{4}\left\{\lambda^{2}\left[J_{\ell}^{\prime}(\lambda a) J_{\ell}^{\prime \prime \prime}(\lambda a)+(-1)^{\ell} I_{\ell}^{\prime}(\lambda a) I_{\ell}^{\prime \prime \prime}(\lambda a)\right]\right. \\
& +\frac{\lambda}{a}\left[J_{\ell}^{\prime}(\lambda a) J_{\ell}^{\prime \prime}(\lambda a)+(-1)^{\ell} I_{\ell}^{\prime}(\lambda a) I_{\ell}^{\prime \prime}(\lambda a)\right] \\
& -\frac{1}{a^{2}}\left[J_{\ell}^{\prime}(\lambda a) J_{\ell}^{\prime}(\lambda a)+(-1)^{\ell} I_{\ell}^{\prime}(\lambda a) I_{\ell}^{\prime}(\lambda a)\right] \\
& +\frac{\ell^{2}(3-v)}{\lambda a^{3}}\left[J_{\ell}^{\prime}(\lambda a) J_{\ell}(\lambda a)+(-1)^{\ell} I_{\ell}(\lambda a) I_{\ell}(\lambda a)\right] \\
& \left.-\frac{\ell^{2}(2-v)}{a^{2}}\left[J_{\ell}^{\prime}(\lambda a) J_{\ell}^{\prime}(\lambda a)+(-1)^{\ell} I_{\ell}^{\prime}(\lambda a) I_{\ell}^{\prime}(\lambda a)\right]\right\}
\end{aligned}
$$

$$
\begin{aligned}
v_{\ell}^{[U]}= & \frac{N}{4}\left\{\left[J_{\ell}^{\prime \prime}(\lambda a) J_{\ell}(\lambda a)+(-1)^{\ell} I_{\ell}^{\prime \prime}(\lambda a) I_{\ell}(\lambda a)\right]\right. \\
& +\frac{v}{\lambda a}\left[J_{\ell}^{\prime}(\lambda a) J_{\ell}(\lambda a)+(-1)^{\ell} I_{\ell}^{\prime}(\lambda a) I_{\ell}(\lambda a)\right] \\
& \left.-\frac{\ell v}{\lambda^{2} a^{2}}\left[J_{\ell}(\lambda a) J_{\ell}(\lambda a)+(-1)^{\ell} I_{\ell}(\lambda a) I_{\ell}(\lambda a)\right]\right\}
\end{aligned}
$$




$$
\begin{aligned}
v_{\ell}^{[\Theta]}= & \frac{N}{4}\left\{\left[J_{\ell}^{\prime \prime}(\lambda a) J_{\ell}^{\prime}(\lambda a)+(-1)^{\ell} I_{\ell}^{\prime \prime}(\lambda a) I_{\ell}^{\prime}(\lambda a)\right]\right. \\
& +\frac{v_{a}}{a}\left[J_{\ell}^{\prime}(\lambda a) J_{\ell}^{\prime}(\lambda a)+(-1)^{\ell} I_{\ell}^{\prime}(\lambda a) I_{\ell}^{\prime}(\lambda a)\right] \\
& \left.-\frac{\ell v}{\lambda a^{2}}\left[J_{\ell}(\lambda a) J_{\ell}^{\prime}(\lambda a)+(-1)^{\ell} I_{\ell}(\lambda a) I_{\ell}^{\prime}(\lambda a)\right]\right\} \\
v_{\ell}^{[M]}= & \frac{N}{4}\left\{\lambda^{2}\left[J_{\ell}^{\prime \prime}(\lambda a) J_{\ell}^{\prime \prime}(\lambda a)+(-1)^{\ell} I_{\ell}^{\prime \prime}(\lambda a) I_{\ell}^{\prime \prime}(\lambda a)\right]\right. \\
& +\frac{\lambda v}{a}\left[J_{\ell}^{\prime}(\lambda a) J_{\ell}^{\prime \prime}(\lambda a)+(-1)^{\ell} I_{\ell}^{\prime}(\lambda a) I_{\ell}^{\prime \prime}(\lambda a)\right] \\
& \left.-\frac{\ell^{2} v^{2}}{a^{2}}\left[J_{\ell}(\lambda a) J_{\ell}^{\prime \prime}(\lambda a)+(-1)^{\ell} I_{\ell}(\lambda a) I_{\ell}^{\prime \prime}(\lambda a)\right]\right\} \\
& +\frac{v}{a} \mu_{\ell}^{[M]}-\frac{\ell^{2} v^{2}}{a^{2}} \lambda_{\ell}^{[M]}
\end{aligned}
$$

$v_{\ell}^{[V]}$

$$
\begin{aligned}
= & \frac{N}{4}\left\{\lambda^{3}\left[J_{\ell}^{\prime \prime \prime}(\lambda a) J_{\ell}^{\prime \prime}(\lambda a)+(-1)^{\ell} I_{\ell}^{\prime \prime \prime}(\lambda a) I_{\ell}^{\prime \prime}(\lambda a)\right]\right. \\
& +\frac{\lambda}{a^{2}}\left[J_{\ell}^{\prime}(\lambda a) J_{\ell}^{\prime \prime}(\lambda a)+(-1)^{\ell} I_{\ell}^{\prime}(\lambda a) I_{\ell}^{\prime \prime}(\lambda a)\right] \\
& +\frac{\lambda^{2}}{a}\left[J_{\ell}^{\prime \prime}(\lambda a) J_{\ell}^{\prime \prime}(\lambda a)+(-1)^{\ell} I_{\ell}^{\prime \prime}(\lambda a) I_{\ell}^{\prime \prime}(\lambda a)\right] \\
& -\frac{\ell^{2}(3-v)}{a^{3}}\left[J_{\ell}(\lambda a) J_{\ell}^{\prime \prime}(\lambda a)+(-1)^{\ell} I_{\ell}(\lambda a) I_{\ell}^{\prime \prime}(\lambda a)\right] \\
& -\frac{\ell^{2} \lambda(2-v)}{a^{2}}\left[J_{\ell}^{\prime}(\lambda a) J_{\ell}^{\prime \prime}(\lambda a)+(-1)^{\ell} I_{\ell}^{\prime}(\lambda a) I_{\ell}^{\prime \prime}(\lambda a)\right]
\end{aligned}
$$

$\delta_{\ell}^{[U]}$

$$
\begin{aligned}
= & \frac{N}{4}\left\{\lambda\left[J_{\ell}^{\prime \prime \prime}(\lambda a) J_{\ell}(\lambda a)+(-1)^{\ell} I_{\ell}^{\prime \prime \prime}(\lambda a) I_{\ell}(\lambda a)\right]\right. \\
& +\frac{1}{a}\left[J_{\ell}^{\prime \prime}(\lambda a) J_{\ell}(\lambda a)+(-1)^{\ell} I_{\ell}^{\prime \prime}(\lambda a) I_{\ell}(\lambda a)\right] \\
& -\frac{1}{\lambda a^{2}}\left[J_{\ell}^{\prime}(\lambda a) J_{\ell}(\lambda a)+(-1)^{\ell} I_{\ell}^{\prime}(\lambda a) I_{\ell}(\lambda a)\right] \\
& +\frac{\ell^{2}(3-v)}{\lambda^{2} a^{3}}\left[J_{\ell}(\lambda a) J_{\ell}(\lambda a)+(-1)^{\ell} I_{\ell}(\lambda a) I_{\ell}(\lambda a)\right] \\
& \left.-\frac{\ell^{2}(2-v)}{\lambda a^{2}}\left[J_{\ell}^{\prime}(\lambda a) J_{\ell}(\lambda a)+(-1)^{\ell} I_{\ell}^{\prime}(\lambda a) I_{\ell}(\lambda a)\right]\right\}
\end{aligned}
$$

$$
\begin{aligned}
& \delta_{\ell}^{[\Theta]} \\
&=\frac{N}{4}\left\{\lambda\left[J_{\ell}^{\prime \prime \prime}(\lambda a) J_{\ell}(\lambda a)+(-1)^{\ell} I_{\ell}^{\prime \prime \prime}(\lambda a) I_{\ell}(\lambda a)\right]\right. \\
&+\frac{1}{a}\left[J_{\ell}^{\prime \prime}(\lambda a) J_{\ell}(\lambda a)+(-1)^{\ell} I_{\ell}^{\prime \prime}(\lambda a) I_{\ell}(\lambda a)\right] \\
&-\frac{1}{\lambda a^{2}}\left[J_{\ell}^{\prime}(\lambda a) J_{\ell}(\lambda a)+(-1)^{\ell} I_{\ell}^{\prime}(\lambda a) I_{\ell}(\lambda a)\right] \\
&+\frac{\ell^{2}(3-v)}{\lambda^{2} a^{3}}\left[J_{\ell}(\lambda a) J_{\ell}(\lambda a)+(-1)^{\ell} I_{\ell}(\lambda a) I_{\ell}(\lambda a)\right] \\
&\left.-\frac{\ell^{2}(2-v)}{\lambda a^{2}}\left[J_{\ell}^{\prime}(\lambda a) J_{\ell}(\lambda a)+(-1)^{\ell} I_{\ell}^{\prime}(\lambda a) I_{\ell}(\lambda a)\right]\right\}
\end{aligned}
$$

$\delta_{\ell}^{[M]}$

$=\frac{N}{4}\left\{\lambda^{3}\left[J_{\ell}^{\prime \prime}(\lambda a) J_{\ell}^{\prime \prime \prime}(\lambda a)+(-1)^{\ell} I_{\ell}^{\prime \prime}(\lambda a) I_{\ell}^{\prime \prime \prime}(\lambda a)\right]\right.$

$+\frac{\lambda^{2} v^{a}}{a}\left[J_{\ell}^{\prime}(\lambda a) J_{\ell}^{\prime \prime \prime}(\lambda a)+(-1)^{\ell} I_{\ell}^{\prime}(\lambda a) I_{\ell}^{\prime \prime \prime}(\lambda a)\right]$

$\left.-\frac{\ell^{2} \lambda v}{a^{2}}\left[J_{\ell}(\lambda a) J_{\ell}^{\prime \prime \prime}(\lambda a)+(-1)^{\ell} I_{\ell}(\lambda a) I_{\ell}^{\prime \prime \prime}(\lambda a)\right]\right\}$

$+\frac{N}{4 a}\left\{\lambda^{2}\left[J_{\ell}^{\prime \prime}(\lambda a) J_{\ell}^{\prime \prime}(\lambda a)+(-1)^{\ell} I_{\ell}^{\prime \prime}(\lambda a) I_{\ell}^{\prime \prime}(\lambda a)\right]\right.$

$+\frac{\lambda v}{a}\left[J_{\ell}^{\prime}(\lambda a) J_{\ell}^{\prime \prime}(\lambda a)-\frac{1}{a^{2}} \mu_{\ell}^{[M]}+\frac{\ell^{2}(3-v)}{a^{3}} \lambda_{\ell}^{[M]}\right.$

$-\frac{\ell^{2}(2-v)}{a^{2}} \mu_{\ell}^{[M]}$

$\delta_{\ell}^{[V]}$

$=\frac{N}{4}\left\{\lambda^{4}\left[J_{\ell}^{\prime \prime \prime}(\lambda a) J_{\ell}^{\prime \prime \prime}(\lambda a)+(-1)^{\ell} I_{\ell}^{\prime \prime \prime}(\lambda a) I_{\ell}^{\prime \prime \prime}(\lambda a)\right]\right.$

$+\frac{\lambda^{2}}{a^{2}}\left[J_{\ell}^{\prime}(\lambda a) J_{\ell}^{\prime \prime \prime}(\lambda a)+(-1)^{\ell} I_{\ell}^{\prime}(\lambda a) I_{\ell}^{\prime \prime \prime}(\lambda a)\right]$

$+\frac{\lambda^{3}}{a}\left[J_{\ell}^{\prime \prime}(\lambda a) J_{\ell}^{\prime \prime \prime}(\lambda a)+(-1)^{\ell} I_{\ell}^{\prime \prime}(\lambda a) I_{\ell}^{\prime \prime \prime}(\lambda a)\right]$

$+\frac{\lambda \ell^{2}(3-v)}{a^{3}}\left[J_{\ell}(\lambda a) J_{\ell}^{\prime \prime \prime}(\lambda a)+(-1)^{\ell} I_{\ell}(\lambda a) I_{\ell}^{\prime \prime \prime}(\lambda a)\right]$

$\left.-\frac{\lambda^{2} \ell^{2}(2-v)}{a^{2}}\left[J_{\ell}^{\prime}(\lambda a) J_{\ell}^{\prime \prime \prime}(\lambda a)+(-1)^{\ell} I_{\ell}^{\prime}(\lambda a) I_{\ell}^{\prime \prime \prime}(\lambda a)\right]\right\}$

$+\frac{N}{4 a}\left\{\lambda^{3}\left[J_{\ell}^{\prime \prime \prime}(\lambda a) J_{\ell}^{\prime \prime}(\lambda a)+(-1)^{\ell} I_{\ell}^{\prime \prime \prime}(\lambda a) I_{\ell}^{\prime \prime}(\lambda a)\right]\right.$

$+\frac{\lambda}{a^{2}}\left[J_{\ell}^{\prime}(\lambda a) J_{\ell}^{\prime \prime}(\lambda a)-\frac{1}{a^{2}} \mu_{\ell}^{[V]}+\frac{\ell^{2}(3-v)}{a^{3}} \lambda_{\ell}^{[V]}\right.$

$-\frac{\ell^{2}(2-v)}{a^{2}} \mu_{\ell}^{[V]}$

Employing the SVD techinque, we can decompose the $[U]$ matrix into

$$
[U]=\Phi \Sigma_{U} \Phi^{-1}=\Phi \operatorname{diag}\left(\lambda_{0}^{[U]}, \lambda_{1}^{[U]}, \lambda_{-1}^{[U]},\right.
$$$$
\left.\cdots, \lambda_{(N-1)}^{[U]}, \lambda_{-(N-1)}^{[U]}, \lambda_{N}^{[U]}\right) \Phi^{-1}
$$ 
where

$$
\begin{aligned}
& \Phi=\frac{1}{\sqrt{2} N} \\
& {\left[\begin{array}{ccccccc} 
\\
1 & 1 & 0 & \cdots & 1 & 0 & 1 \\
1 & \cos \left(\frac{2 \pi}{2 N}\right) & \sin \left(\frac{2 \pi}{2 N}\right) & \cdots & \cos \left(\frac{2 \pi(N-1)}{2 N}\right) & \sin \left(\frac{2 \pi(2 N-1)}{2 N}\right) & \cos \left(\frac{2 \pi N}{2 N}\right) \\
1 & \cos \left(\frac{4 \pi}{2 N}\right) & \sin \left(\frac{4 \pi}{2 N}\right) & \cdots & \cos \left(\frac{4 \pi(N-1)}{2 N}\right) & \sin \left(\frac{4 \pi(N-1)}{2 N}\right) & \cos \left(\frac{4 \pi N}{2 N}\right) \\
\vdots & \vdots & \vdots & \ddots & \vdots & \vdots \\
1 & \cos \left(\frac{2 \pi(2 N-2)}{2 N}\right) & \sin \left(\frac{2 \pi(2 N-2)}{2 N}\right) & \cdots & \cos \left(\frac{\pi(4 N-4)(N-1)}{2 N}\right) & \sin \left(\frac{\pi(4 N-4)(N-1)}{2 N}\right) & \cos \left(\frac{\pi(4 N-4)(N)}{2 N}\right) \\
1 & \cos \left(\frac{2 \pi(2 N-1)}{2 N}\right) & \sin \left(\frac{2 \pi(2 N-1)}{2 N}\right) & \cdots & \cos \left(\frac{\pi(4 N-2)(N-1)}{2 N}\right) & \sin \left(\frac{\pi(4 N-2)(N-1)}{2 N}\right) & \cos \left(\frac{\pi(4 N-2)(N)}{2 N}\right) \\
& & & & & & \vdots
\end{array}\right]_{2 N \times 2 N}}
\end{aligned}
$$

Similarly, the other fifteen matrices, $[\Theta],[M],[V]$, $\left[U_{\theta}\right],\left[\Theta_{\theta}\right],\left[M_{\theta}\right],\left[V_{\theta}\right],\left[U_{m}\right],\left[\Theta_{m}\right],\left[M_{m}\right],\left[V_{m}\right],\left[U_{v}\right]$, $\left[\Theta_{v}\right],\left[M_{v}\right]$ and $\left[V_{v}\right]$ can be decomposed by changing the diagonal element with the corresponding eigenvalue in Eqs. (3)-(17), respectively. Eq. (20) can be rewritten as

$$
\left[S M_{1}^{c}\right]=\left[\begin{array}{cc}
\Phi \Sigma_{U} \Phi^{-1} & \Phi \Sigma_{\Theta} \Phi^{-1} \\
\Phi \Sigma_{U_{\theta}} \Phi^{-1} & \Phi \Sigma_{\Theta_{\theta}} \Phi^{-1}
\end{array}\right]_{4 N \times 4 N}
$$

Eq. (45) can be reformulated into

$$
\left[S M_{1}^{c}\right]=\left[\begin{array}{cc}
\Phi & 0 \\
0 & \Phi
\end{array}\right]\left[\begin{array}{cc}
\Sigma_{U} & \Sigma_{\Theta} \\
\Sigma_{U_{\theta}} & \Sigma_{\Theta_{\theta}}
\end{array}\right]\left[\begin{array}{cc}
\Phi & 0 \\
0 & \Phi
\end{array}\right]^{-1}
$$

Since $\Phi$ is orthogonal $\left(\operatorname{det}|\Phi|=\operatorname{det}\left|\Phi^{-1}\right|=1\right)$, the determinant of $\left[S M_{1}^{c}\right]_{4 N \times 4 N}$ is

$$
\begin{aligned}
\operatorname{det}\left[S M_{1}^{c}\right] & =\operatorname{det}\left[\begin{array}{cc}
\Sigma_{U} & \Sigma_{\Theta} \\
\Sigma_{U_{\theta}} & \Sigma_{\Theta_{\theta}}
\end{array}\right] \\
& =\prod_{\ell=-(N+1)}^{N}\left(\lambda_{\ell}^{[U]} \mu_{\ell}^{[\Theta]}-\lambda_{\ell}^{[\Theta]} \mu_{\ell}^{[U]}\right)
\end{aligned}
$$

By employing Eqs. (27), (28), (31) and (32), we have

$$
\begin{aligned}
& \operatorname{det}\left[S M_{1}^{c}\right] \\
& =\prod_{\ell=-(N-1)}^{N} \frac{(-1)^{\ell} N^{2}}{16 \lambda^{2}}\left\{J_{\ell}^{\prime}(\lambda a) I_{\ell}(\lambda a)-J_{\ell}(\lambda a) I_{\ell}^{\prime}(\lambda a)\right\} \\
& \cdot\left[J_{\ell}^{\prime}(\lambda a) I_{\ell}(\lambda a)-J_{\ell}(\lambda a) J_{\ell}^{\prime}(\lambda a)\right]
\end{aligned}
$$

for the clamped case. By using the differential property of Bessel function, Eq. (48) can be reduced to

$$
\begin{aligned}
& \left\{J_{\ell+1}(\lambda a) I_{\ell}(\lambda a)+J_{\ell}(\lambda a) I_{\ell+1}(\lambda a)\right\}\left[J_{\ell+1}(\lambda a) I_{\ell}(\lambda a)\right. \\
& \left.+J_{\ell}(\lambda a) I_{\ell+1}(\lambda a)\right]=0 \\
& \quad \ell=0, \pm 1, \pm 2, \ldots, \pm(N-1), N
\end{aligned}
$$

The eigenequation in Eq. (49) is collected together to

$$
\left(J_{\ell}(\lambda a) I_{\ell+1}(\lambda a)+J_{\ell+1}(\lambda a) I_{\ell}(\lambda a)\right)^{2}=0
$$

The true eigenequation matches the exact solution well (Leissa, 1969). The present approach can be easily applied to the simply-supported and free plates.

For the simply-supported plate $(u=0$ and $m=0)$, Eq. (20) becomes

$$
\left[S M_{1}^{s}\right]=\left[\begin{array}{cc}
U & \Theta \\
U_{m} & \Theta_{m}
\end{array}\right]_{4 N \times 4 N}
$$

where the superscript " $s$ " denotes the simply-supported case. Similarly, we have

$$
\operatorname{det}\left[S M_{1}^{s}\right]=\prod_{\ell=-(N-1)}^{N}\left(\lambda_{\ell}^{[U]} v_{\ell}^{[\Theta]}-\lambda_{\ell}^{[\Theta]} v_{\ell}^{[U]}\right)
$$

By employing Eqs. (27), (29), (35) and (37), we have 


$$
\begin{aligned}
& \operatorname{det}\left[S M_{1}^{s}\right] \\
& =\prod_{-(N-1)}^{N} \frac{(-1)^{\ell} N^{2}}{16 \lambda^{2} a}\left[J_{\ell+1}(\lambda a) I_{\ell}(\lambda a)+J_{\ell}(\lambda a) I_{\ell+1}(\lambda a)\right] \\
& \left\{(-1+v)\left(J_{\ell+1}(\lambda a) I_{\ell}(\lambda a)+J_{\ell}(\lambda a) I_{\ell+1}(\lambda a)\right)\right. \\
& \left.+2 \lambda a J_{\ell}(\lambda a) I_{\ell}(\lambda a)\right\} \\
& \quad \quad=0, \pm 1, \pm 2, \ldots, \pm(N-1), N
\end{aligned}
$$

Eq. (53) indicates that the derived eigenequation is

$$
\begin{aligned}
& {\left[J_{\ell+1}(\lambda a) I_{\ell}(\lambda a)+J_{\ell}(\lambda a) I_{\ell+1}(\lambda a)\right]} \\
& \left\{(-1+v)\left(J_{\ell+1}(\lambda a) I_{\ell}(\lambda a)+J_{\ell}(\lambda a) I_{\ell+1}(\lambda a)\right)\right. \\
& \left.+2 \lambda a J_{\ell}(\lambda a) I_{\ell}(\lambda a)\right\}=0
\end{aligned}
$$

The possible eigenequation is either the former part

$$
\left[J_{\ell+1}(\lambda a) I_{\ell}(\lambda a)+J_{\ell}(\lambda a) I_{\ell+1}(\lambda a)\right]=0
$$

or the latter part

$$
\begin{aligned}
& (-1+v)\left(J_{\ell+1}(\lambda a) I_{\ell}(\lambda a)+J_{\ell}(\lambda a) I_{\ell+1}(\lambda a)\right) \\
& +2 \lambda a J_{\ell}(\lambda a) I_{\ell}(\lambda a)=0
\end{aligned}
$$

Equation (56) can be simplified to

$$
\frac{J_{\ell+1}(\lambda a)}{J_{\ell}(\lambda a)}+\frac{I_{\ell+1}(\lambda a)}{I_{\ell}(\lambda a)}=\frac{2 \lambda a}{1-v}
$$

Eq. (57) is found to be the true eigenequation of the simply-supported plate after comparing with the exact solution (Leissa, 1969), while Eq. (55) is the spurious one, which is exactly the true eigenequation of the clamped plate.

For the free plate ( $m=0$ and $v=0$ ), Eq. (20) becomes

$$
\left[S M_{1}^{f}\right]=\left[\begin{array}{cc}
U_{m} & \Theta_{m} \\
U_{v} & \Theta_{v}
\end{array}\right]_{4 N \times 4 N}
$$

where the superscript " $f$ " denotes the free case. Similarly, we have

$$
\operatorname{det}\left[S M_{1}^{f}\right]=\prod_{\ell=-(N-1)}^{N}\left(v_{\ell}^{[U]} \delta_{\ell}^{[\Theta]}-v_{\ell}^{[\Theta]} \delta_{\ell}^{[U]}\right)
$$

By employing Eqs. (35), (36), (39) and (40), we have

$$
\begin{aligned}
& \operatorname{det}\left[S M_{1}^{f}\right] \\
& =\prod_{\ell=-(N-1)}^{N} \frac{(-1)^{\ell} N^{2}}{16 \lambda^{2} a^{4}}\left[J_{\ell+1}(\lambda a) I_{\ell}(\lambda a)+J_{\ell}(\lambda a) I_{\ell+1}(\lambda a)\right] \\
& \\
& \left\{( \ell ^ { 2 } ( \ell ^ { 2 } - 1 ) ( - 1 + v ) ^ { 2 } + \lambda ^ { 4 } a ^ { 4 } ) \left(J_{\ell+1}(\lambda a) I_{\ell}(\lambda a)\right.\right. \\
& \left.+J_{\ell}(\lambda a) I_{\ell+1}(\lambda a)\right)+2 \ell \lambda^{2} a^{2}(1-\ell(v-1)) \\
& +\left(J_{\ell+1}(\lambda a) I_{\ell}(\lambda a)-J_{\ell}(\lambda a) I_{\ell+1}(\lambda a)\right) \\
& +\lambda a(-1+v)\left(2 \lambda^{2} a^{2} J_{\ell+1}(\lambda a) I_{\ell+1}(\lambda a)\right) \\
& \left.+4 \ell^{2}(-1+\ell) J_{\ell}(\lambda a) I_{\ell}(\lambda a)\right\}
\end{aligned}
$$

According to the zero determinant in Eq. (60), the derived eigenequation is

$$
\begin{aligned}
& {\left[J_{\ell+1}(\lambda a) I_{\ell}(\lambda a)+J_{\ell}(\lambda a) I_{\ell+1}(\lambda a)\right]} \\
& \left\{( \ell ^ { 2 } ( \ell ^ { 2 } - 1 ) ( - 1 + v ) ^ { 2 } + \lambda ^ { 4 } a ^ { 4 } ) \left(J_{\ell+1}(\lambda a) I_{\ell}(\lambda a)\right.\right. \\
& \left.+J_{\ell}(\lambda a) I_{\ell+1}(\lambda a)\right)+2 \ell \lambda^{2} a^{2}(1-\ell(v-1)) \\
& \cdot\left(J_{\ell+1}(\lambda a) I_{\ell}(\lambda a)-J_{\ell}(\lambda a) I_{\ell+1}(\lambda a)\right) \\
& +\lambda a(-1+v)\left(2 \lambda^{2} a^{2} J_{\ell+1}(\lambda a) I_{\ell+1}(\lambda a)\right) \\
& \left.+4 \ell^{2}(-1+\ell) J_{\ell}(\lambda a) I_{\ell}(\lambda a)\right\}=0 \\
& \ell=0, \pm 1, \pm 2, \cdots, \pm N
\end{aligned}
$$

The possible eigenequation can be either the former part

$$
J_{\ell+1}(\lambda a) I_{\ell}(\lambda a)+J_{\ell}(\lambda a) I_{\ell+1}(\lambda a)=0
$$

or the latter part

$$
\begin{aligned}
& \left(\ell^{2}\left(\ell^{2}-1\right)(-1+v)^{2}+\lambda^{4} r^{4}\right)\left(J_{\ell+1}(\lambda a) I_{\ell}(\lambda a)\right. \\
& \left.+J_{\ell}(\lambda a) I_{\ell+1}(\lambda a)\right)+2 \ell \lambda^{2} a^{2}(1-\ell(v-1)) \\
& \cdot\left(J_{\ell+1}(\lambda a) I_{\ell}(\lambda a)-J_{\ell}(\lambda a) I_{\ell+1}(\lambda a)\right) \\
& +\lambda a(-1+v)\left(2 \lambda^{2} a^{2} J_{\ell+1}(\lambda a) I_{\ell+1}(\lambda a)\right) \\
& \left.+4 \ell^{2}(-1+\ell) J_{\ell}(\lambda a) I_{\ell}(\lambda a)\right\}=0
\end{aligned}
$$

It is interesting to find that spurious eigenequations in Eqs. (55)and (62) are all imbedded in the zero determinant for the clamped, simply-supported and free plates. Therefore, Eq. (49) can be understood that the true and spurious eigenequations are the same for the clamped plate using $U$ and $\Theta$ kernels. If the clamped 
plate is solved by using the six formulations (a) $U, \Theta$, (b) $U, M$, (c) $U, V$, (d) $\Theta, M$, (e) $\Theta, V$ and (f) $M$, $V$, the true eigenvalues are found by detecting the zero determinant in Fig. 2 and spurious eigenvalues appear in Fig. 3. The true and spurious eigenvalues satisfy the true and spurious eigenequation obtained by the discrete system in Table 1. Once the formulation is chosen, e.g., $U$ and $\Theta$ kernels, the three cases, clamped, simply-supported and free plates can be solved. Figs. 4(a), 4(b) and 4(c) show the true eigenvalues depend on the boundary conditions. Figs. 4(d), 4(e) and 4(f) indicate that spurious eigenvalues are the same since the same $U$ and $\Theta$ formulation is adopted.

It is noted that the true eigenequation of Eq. (63) for free plate is not the same as in Leissa's book (Leissa, 1969) as shown in Table 2. However, the eigenvalues obtained by the present approach match well with Leissa's numerical data. Based on the eigenequation in the Leissa book, we can not obtain the same eigenvalues in his book, i.e., the numerical data are not consistent with his closed-form eigenequation. Maybe, the eigenequation was wrongly printed in Leissa's book. Since the $P$ and $Q$ kernels can be chosen from the four kernels in Eqs. (3)-(6), six ( $\left.C_{2}^{4}\right)$ meshless formulations can be derived. The spurious eigenequations are embedded in the six formulations for any boundary condition of plate problem as shown in Table 1. No matter what formulation is adopted, the same true eigenequation is derived as shown in Table 1 for any one of the clamped, simply-supported and free plates.

\section{EXTRACTION OF THE TRUE EIGENEQUATIONS BY USING SVD UPDATING}

In order to sort out the true eigenvalues in the discrete system, the SVD updating technique is utilized. For the clamped circular plate, we can select $U$ and $\Theta$ kernels for $P$ and $Q$ to obtain Eq. (19). By adopting $P$ and $Q$ as $M$ and $V$ kernels, Eq. (19) becomes

$$
\left[S M_{2}^{c}\right]\left\{\begin{array}{l}
\phi^{\prime} \\
\psi^{\prime}
\end{array}\right\}=\left[\begin{array}{cc}
M & V \\
M_{\theta} & V_{\theta}
\end{array}\right]\left\{\begin{array}{l}
\phi^{\prime} \\
\psi^{\prime}
\end{array}\right\}=\{0\}
$$

where $\phi^{\prime}$ and $\psi^{\prime}$ are the corresponding unknown densities. By using the relation in the degenerate kernels between the direct and the indirect method (Chen et al., 2002), the SVD updating term to extract out the true eigenequation (for the direct method) is equivalent to the SVD updating document (for the indirect method). We have

$$
[C]=\left[\begin{array}{l}
\left(S M_{1}^{c}\right)^{T} \\
\left(S M_{2}^{c}\right)^{T}
\end{array}\right]
$$

where the superscript " $T$ " denotes the transpose. Since the eigenequation is nontrival, the rank of the matrix $[C]$ must be smaller than $4 N$. At least, one of the $4 N$ singular values for the matrix $[C]$ must be zero. By employing the property of Eq. (43), the $[C]$ matrix can be written as

$$
\begin{aligned}
{[C]=} & {\left[\begin{array}{llll}
\Phi & 0 & 0 & 0 \\
0 & \Phi & 0 & 0 \\
0 & 0 & \Phi & 0 \\
0 & 0 & 0 & \Phi
\end{array}\right]\left[\begin{array}{cc}
\Sigma_{U} & \Sigma_{U_{\theta}} \\
\Sigma_{\Theta} & \Sigma_{\Theta_{\theta}} \\
\Sigma_{M} & \Sigma_{M_{\theta}} \\
\Sigma_{V} & \Sigma_{V_{\theta}}
\end{array}\right]_{8 N \times 4 N} } \\
& \cdot\left[\begin{array}{cc}
\Phi^{-1} & 0 \\
0 & \Phi^{-1}
\end{array}\right]
\end{aligned}
$$

Based on the equivalence between the SVD technique and the least-squares method in mathematical essence, the least squares form leads to

$$
[C]^{T}[C]=\left[\begin{array}{cc}
\Phi & 0 \\
0 & \Phi
\end{array}\right][D]_{4 N \times 4 N}\left[\begin{array}{cc}
\Phi^{-1} & 0 \\
0 & \Phi^{-1}
\end{array}\right]
$$

where

$$
[D]=\left[\begin{array}{cccc}
\Sigma_{U} & \Sigma_{\Theta} & \Sigma_{M} & \Sigma_{V} \\
\Sigma_{U_{\theta}} & \Sigma_{\Theta_{\theta}} & \Sigma_{M_{\theta}} & \Sigma_{V_{\theta}}
\end{array}\right]\left[\begin{array}{cc}
\Sigma_{U} & \Sigma_{U_{\theta}} \\
\Sigma_{\Theta} & \Sigma_{\Theta_{\theta}} \\
\Sigma_{M} & \Sigma_{M_{\theta}} \\
\Sigma_{V} & \Sigma_{V_{\theta}}
\end{array}\right]
$$

The zero determinant of the matrix $[C]^{T}[C]$ indicates the existence of a nontrival eigensolution. Since $\Phi$ is orthogonal $\left(\operatorname{det}|\Phi|=\operatorname{det}\left|\Phi^{-1}\right|=1\right)$, the zero determinant of the matrix $[C]^{T}[C]$ implies the zero determinant of the matrix $[D]$. After simplifying the determinant of the matrix $[D]$, we have

$$
\begin{aligned}
& \operatorname{det}[D] \\
& =\prod_{\ell=-(N-1)}^{N}\left[\left(\lambda_{\ell}^{[U]} \mu_{\ell}^{[\Theta]}-\mu_{\ell}^{\left[U_{\theta}\right]} \lambda_{\ell}^{[\Theta]}\right)^{2}\right. \\
& \quad+\left(\lambda_{\ell}^{[U]} \mu_{\ell}^{[M]}-\mu_{\ell}^{[U]} \lambda_{\ell}^{[M]}\right)^{2}+\left(\lambda_{\ell}^{[U]} \mu_{\ell}^{[V]}-\mu_{\ell}^{[U]} \lambda_{\ell}^{[V]}\right)^{2} \\
& \quad+\left(\lambda_{\ell}^{[\Theta]} \mu_{\ell}^{[M]}-\mu_{\ell}^{[\Theta]} \lambda_{\ell}^{[M]}\right)^{2}+\left(\lambda_{\ell}^{[\Theta]} \mu_{\ell}^{[V]}-\mu_{\ell}^{[\Theta]} \lambda_{\ell}^{[V]}\right)^{2} \\
& \left.\quad+\left(\lambda_{\ell}^{[M]} \mu_{\ell}^{[V]}-\mu_{\ell}^{[M]} \lambda_{\ell}^{[V]}\right)^{2}\right] .
\end{aligned}
$$

The only possibility for the zero determinant of $[D]$ is the six terms $\left(\lambda_{\ell}^{[U]} \mu_{\ell}^{[\Theta]}-\mu_{\ell}^{\left[U_{\theta}\right]} \lambda_{\ell}^{[\Theta]}\right),\left(\lambda_{\ell}^{[U]} \mu_{\ell}^{[M]}-\right.$ $\left.\mu_{\ell}^{[U]} \lambda_{\ell}^{[M]}\right),\left(\lambda_{\ell}^{[U]} \mu_{\ell}^{[V]}-\mu_{\ell}^{[U]} \lambda_{\ell}^{[V]}\right),\left(\lambda_{\ell}^{[\Theta]} \mu_{\ell}^{[M]}-\mu_{\ell}^{[\Theta]} \lambda_{\ell}^{[M]}\right)$, $\left(\lambda_{\ell}^{[\Theta]} \mu_{\ell}^{[V]}-\mu_{\ell}^{[\Theta]} \lambda_{\ell}^{[V]}\right)$ and $\left(\lambda_{\ell}^{[M]} \mu_{\ell}^{[V]}-\mu_{\ell}^{[M]} \lambda_{\ell}^{[V]}\right)$ to be zeros at the same time for the same $\ell$. Here we can find that the six zero terms imply six different spurious eigenequations as shown in Table 1 , and the same true 


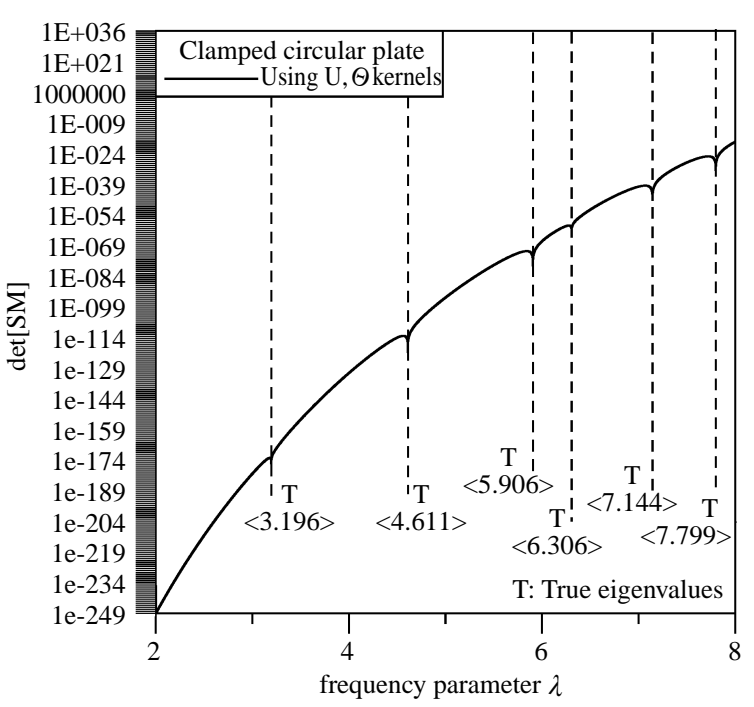

(a)

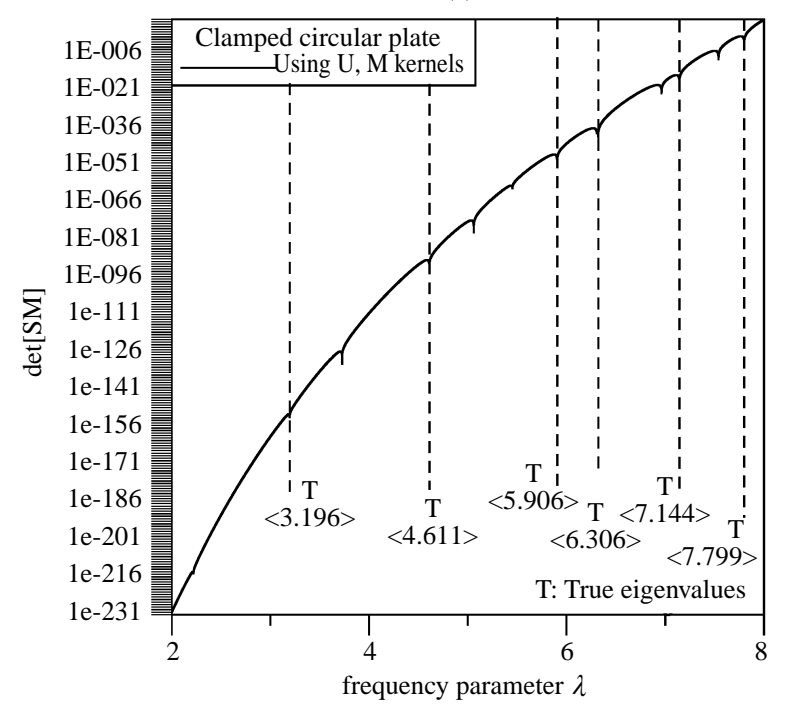

(b)

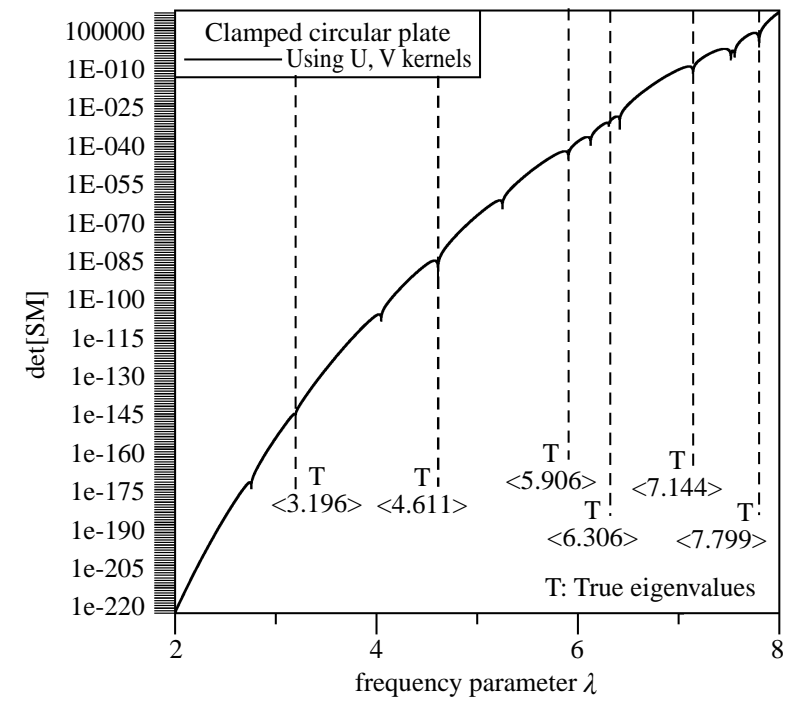

(c)

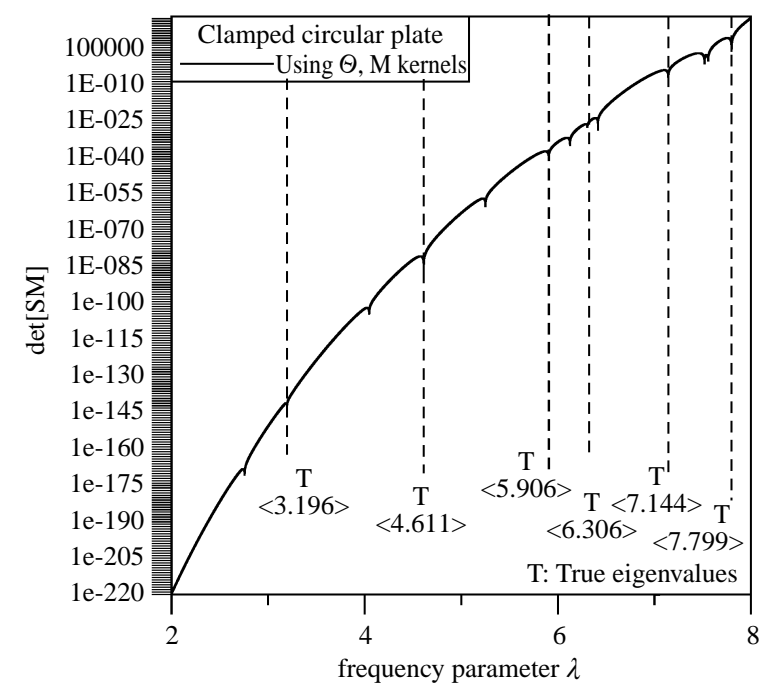

(d)

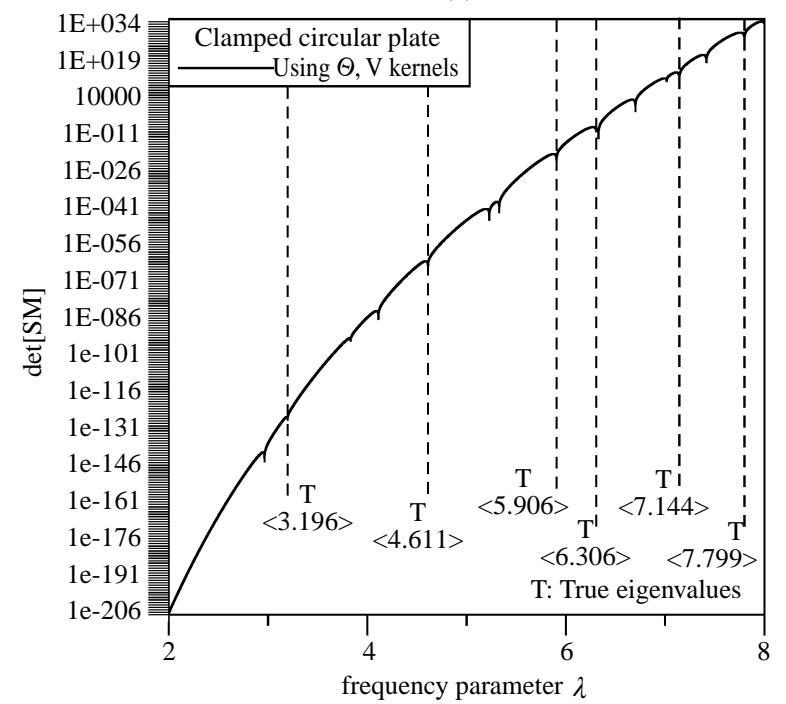

(e)

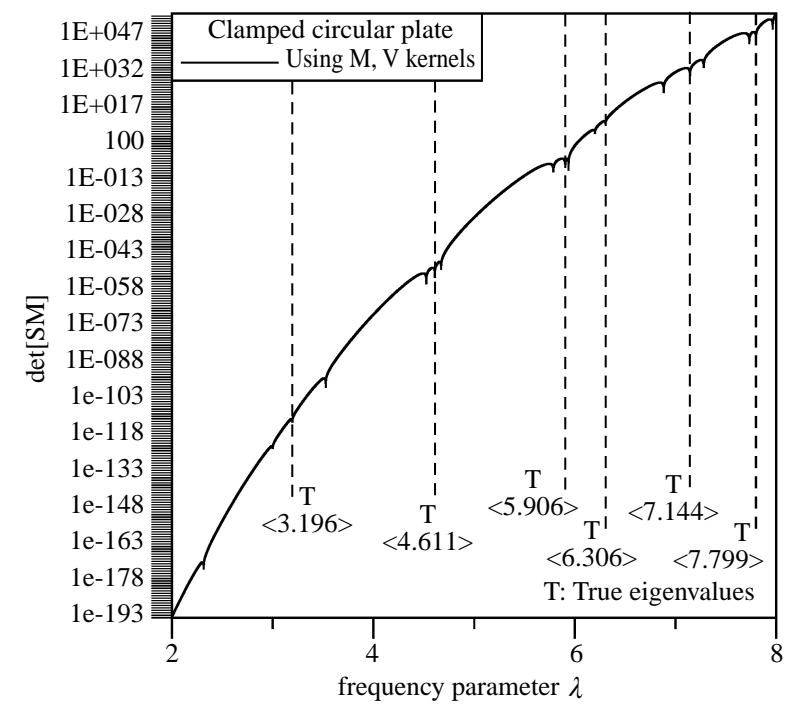

(f)

Fig. 2 Logarithm curve for the $\operatorname{det}[S M]$ versus frequency parameter $\lambda$ for the true eigenvalues of clamped circular plate using different kernels. (a) using $U$ and $\Theta$ kernels; (b) using $U$ and $M$ kernels; (c) using $U$ and $V$ kernels; (d) using $\Theta$ and $M$ kernels; (e) using $\Theta$ and $V$ kernels; (f) using $M$ and $V$ kernels 


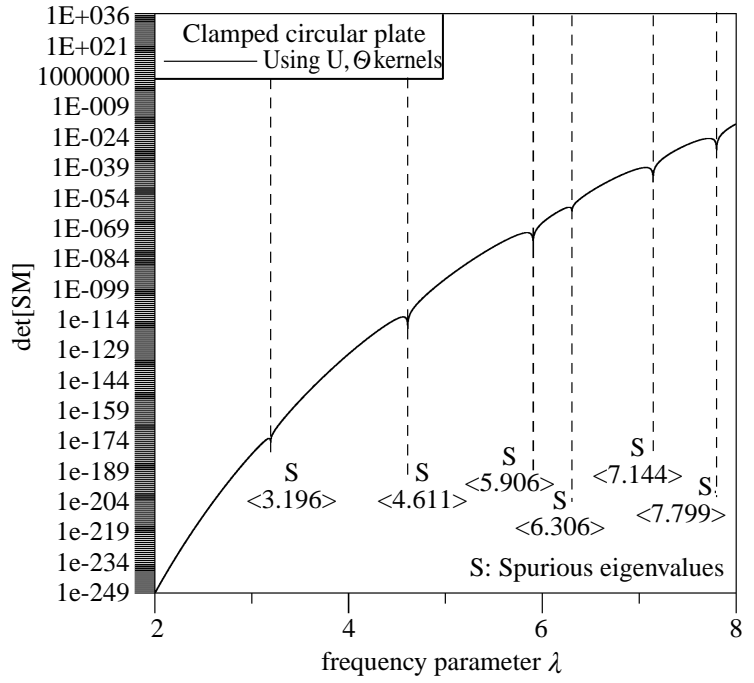

(a)

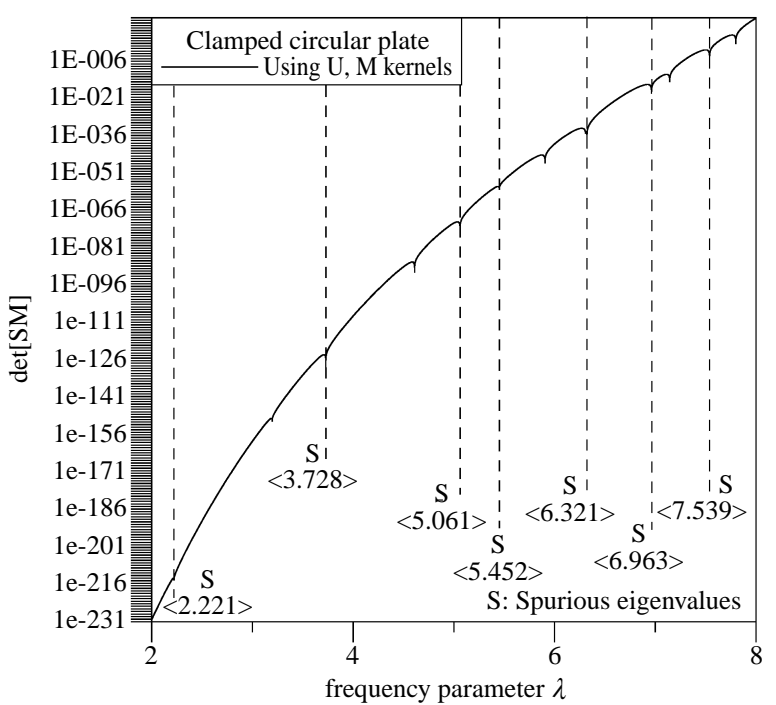

(b)

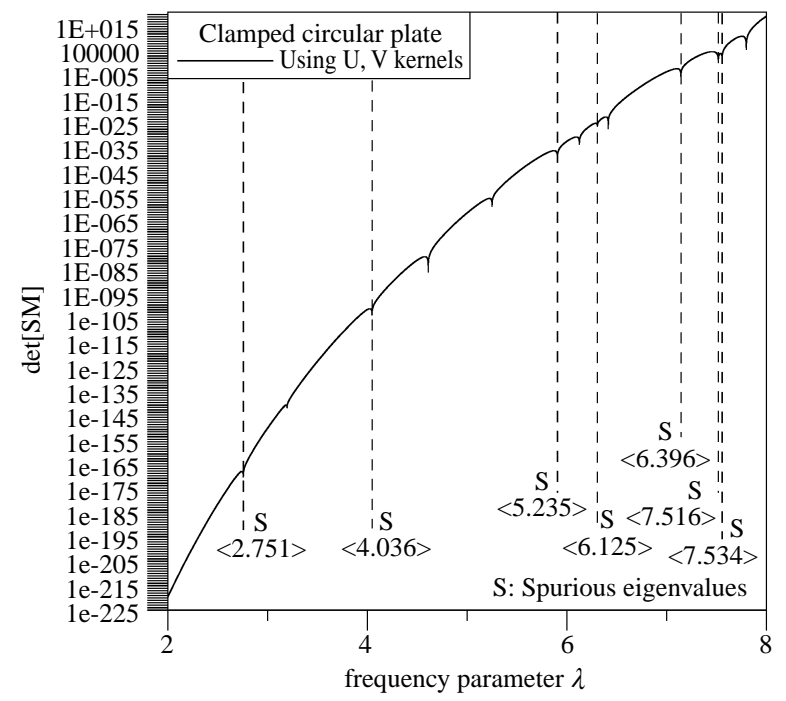

(c)

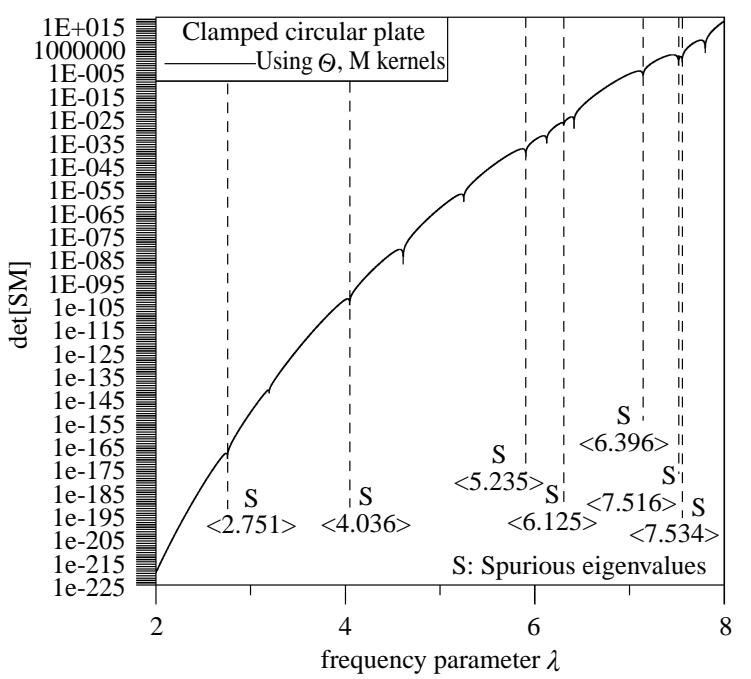

(d)

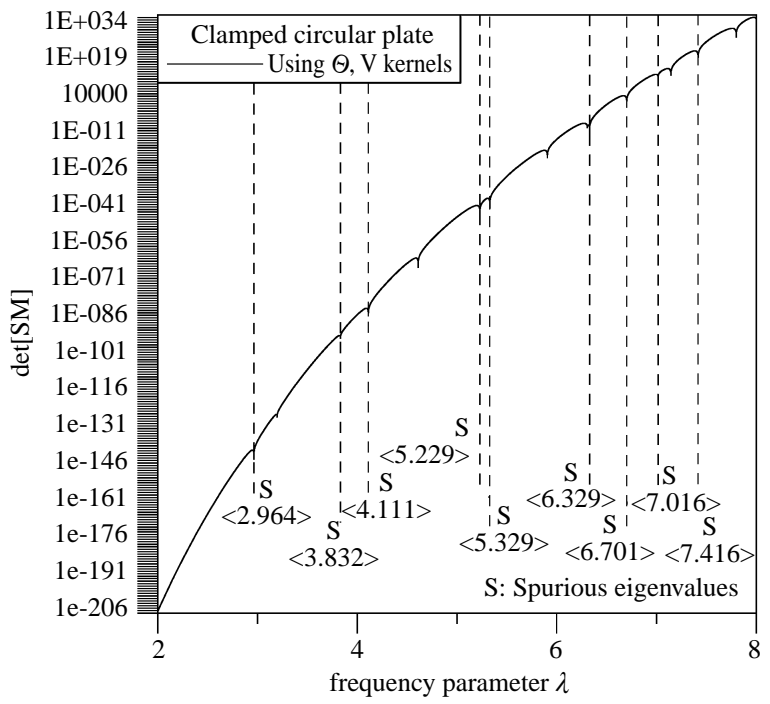

(e)

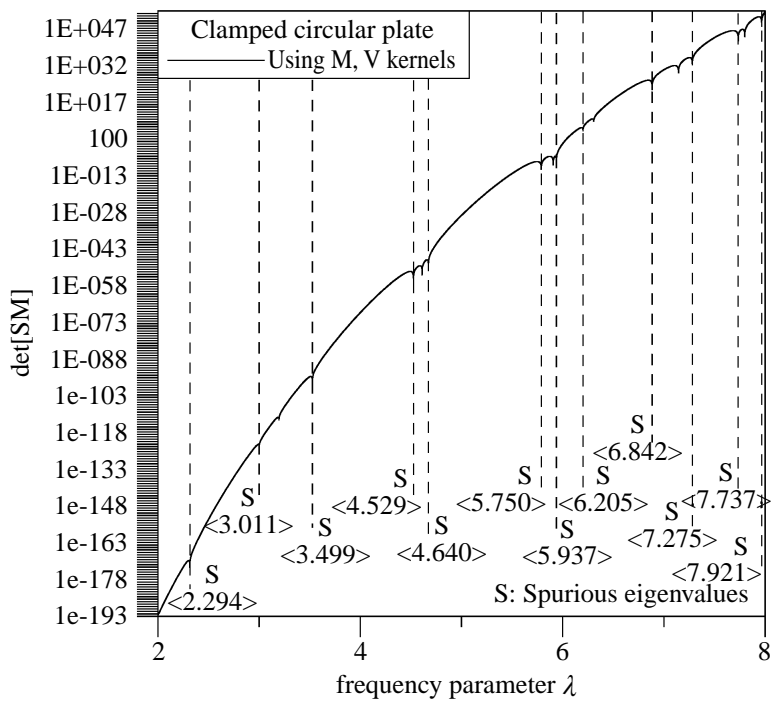

(f)

Fig. 3 Logarithm curve for the $\operatorname{det}[S M]$ versus frequency parameter $\lambda$ for the spurious eigenvalues of clamped circular plate using different kernels. (a) using $U$ and $\Theta$ kernels;(b) using $U$ and $M$ kernels; (c) using $U$ and $V$ kernels; (d) using $\Theta$ and $M$ kernels; (e) using $\Theta$ and $V$ kernels; (f) using $M$ and $V$ kernels 


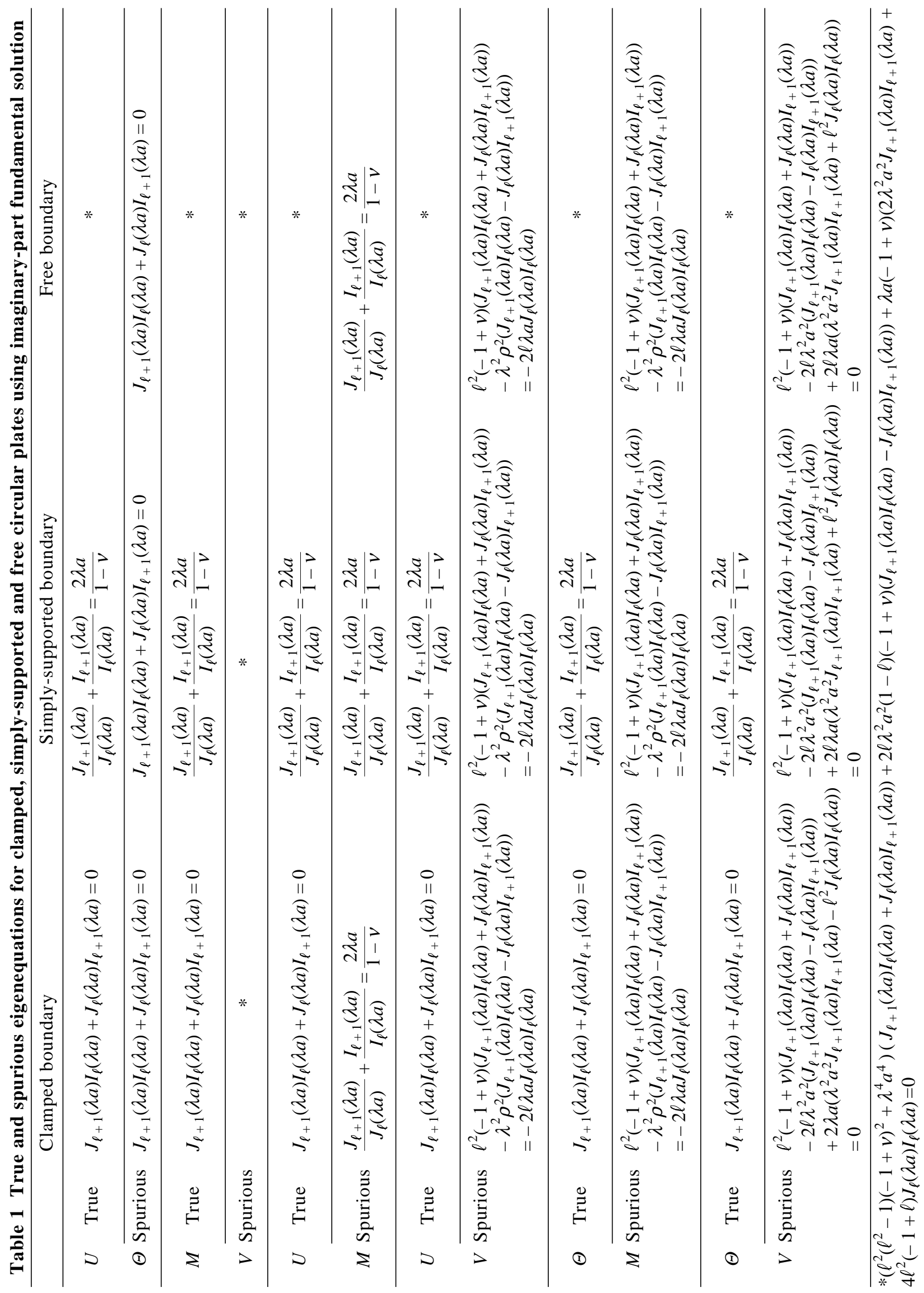




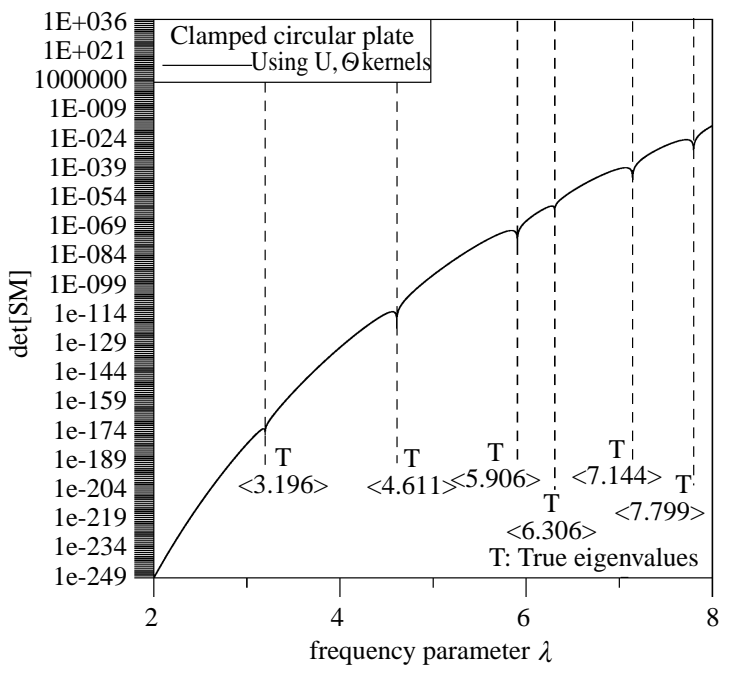

(a)

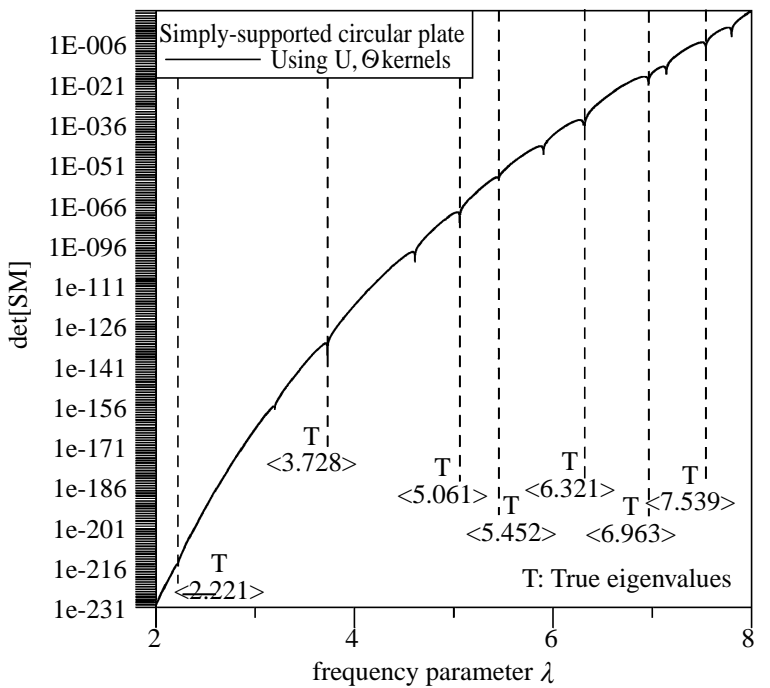

(b)

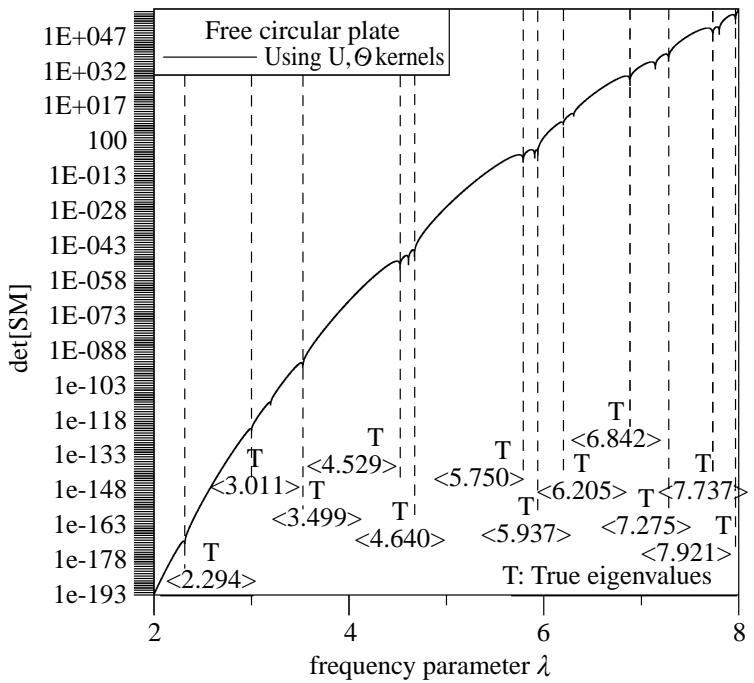

(c)

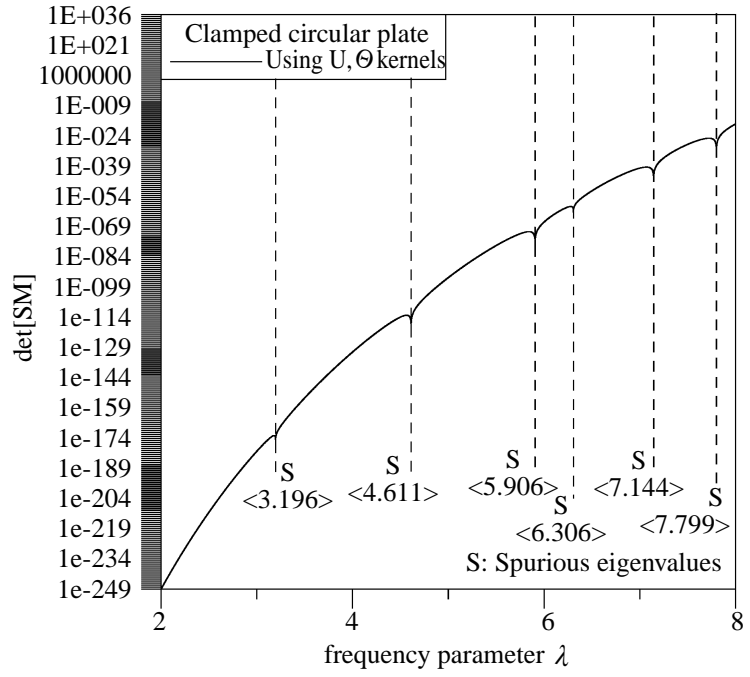

(d)

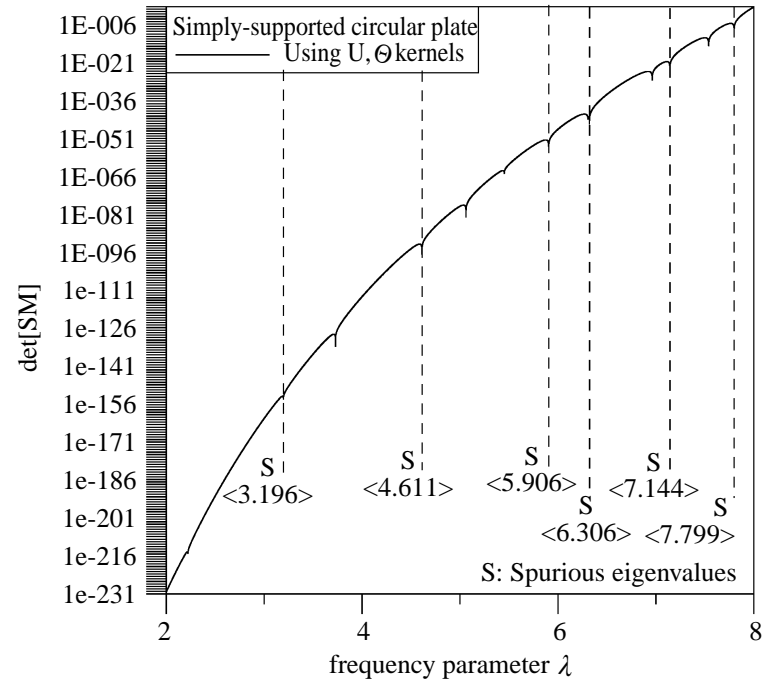

(e)

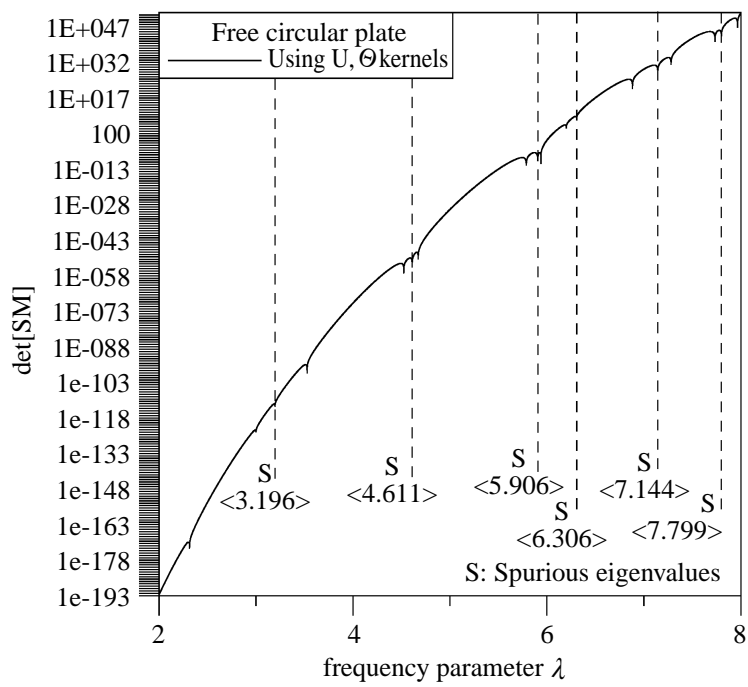

(f)

Fig. 4 Logarithm curve for the $\operatorname{det}[S M]$ versus frequency parameter $\lambda$ for the three boundary conditions using $U$ and $\Theta$ kernels. (a) true eigenvalues of the clamped plate; (b) true eigenvalues of the simply-supported plate; (c) true eigenvalues of the free plate; (d) spurious eigenvalues of the clamped plate; (e) spurious eigenvalues of the simply-supported plate; (f) spurious eigenvalues of the free plate 
Table 2 True eigenequations for a circular plate $(a=1)$

\begin{tabular}{|c|c|c|}
\hline & & True eigenequations for circular plate \\
\hline \multirow{2}{*}{$\begin{array}{l}\text { Clamped } \\
\text { plate }\end{array}$} & Leissa (1969) & $J_{\ell+1}(\lambda) I_{\ell}(\lambda)+J_{\ell}(\lambda) I_{\ell+1}(\lambda)=0$ \\
\hline & Present method & $J_{\ell+1}(\lambda) I_{\ell}(\lambda)+J_{\ell}(\lambda) I_{\ell+1}(\lambda)=0$ \\
\hline \multirow{2}{*}{$\begin{array}{l}\text { Simply- } \\
\text { supported } \\
\text { plate }\end{array}$} & Leissa (1969) & $\frac{J_{\ell+1}(\lambda)}{J_{\ell}(\lambda)}+\frac{I_{\ell+1}(\lambda)}{I_{\ell}(\lambda)}=\frac{2 \lambda}{(1-v)}$ \\
\hline & Present method & $\frac{J_{\ell+1}(\lambda)}{J_{\ell}(\lambda)}+\frac{I_{\ell+1}(\lambda)}{I_{\ell}(\lambda)}=\frac{2 \lambda}{(1-v)}$ \\
\hline \multirow{3}{*}{ Free plate } & Leissa (1969) & 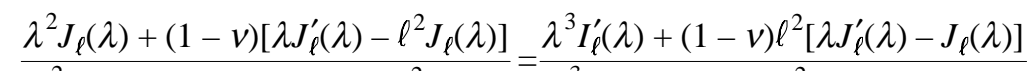 \\
\hline & Leissa $(1909)$ & $\overline{\lambda^{2} I_{\ell}(\lambda)-(1-v)\left[\lambda I_{\ell}^{\prime}(\lambda)-\ell^{2} I_{\ell}(\lambda)\right]}=\overline{\lambda^{3} I_{\ell}^{\prime}(\lambda)-(1-v) \ell^{2}\left[\lambda I_{\ell}^{\prime}(\lambda)-I_{\ell}(\lambda)\right]}$ \\
\hline & Present method & $\begin{array}{l}\left(\ell^{2}\left(\ell^{2}-1\right)(-1+v)^{2}+\lambda^{4}\right)\left(J_{\ell+1}(\lambda) I_{\ell}(\lambda)+J_{\ell}(\lambda) I_{\ell+1}(\lambda)\right) \\
+2 \ell \lambda^{2}(1-\ell)(-1+v)\left(J_{\ell+1}(\lambda) I_{\ell}(\lambda)-J_{\ell}(\lambda) I_{\ell+1}(\lambda)\right) \\
+\lambda(-1+v)\left(2 \lambda^{2} J_{\ell+1}(\lambda) I_{\ell+1}(\lambda)+4 \ell^{2}(-1+\ell) J_{\ell}(\lambda) I_{\ell}(\lambda)=0\right.\end{array}$ \\
\hline
\end{tabular}

where $\ell=0, \pm 1, \pm 2, \pm 3, \cdots$

eigenequation for the clamped case. The only possibility for the zero determinant of $[D]$ is that the common term (true eigenequation) must be zero, i.e.,

$$
\left\{I_{\ell+1}(\lambda a) J_{\ell}(\lambda a)+J_{\ell+1}(\lambda a) I_{\ell}(\lambda a)\right\}=0
$$

This indicates that only the true eigenvalues of the clamped circular plate are imbedded in the SVD updating matrix. The result matches well with Eq. (50) in the discrete system.

\section{CONCLUSIONS}

Meshless formulations have been derived for the eigenproblem of plate vibration. For a circular plate, the true and spurious eigenequations were derived analytically by using the degenerate kernel and circulants. Since any two combinations of the four types of potentials (single, double, triple and quadruple) for solution representation can be chosen, six $\left(C_{2}^{4}\right)$ options were considered. The spurious eigenequation only depends on the adopted kernel functions, while the true eigenequation is relevant to the specified boundary condition (BC). All the true eigenequations for different $\mathrm{BCs}$ and spurious eigenequations in the six formulations are shown in Tables 1 and 2. By using the SVD updating technique, we can extract the true eigenequation. Three cases, clamped, simply-supported and free plates, were demonstrated analytically and numerically to show the validity of the present method. Although only circular plates were treated in the present paper, the same algorithm in the discrete system can be applied to solve arbitrary-shaped plates numerically without any difficulty. Nevertheless, mathematical derivation in both continuous and discrete systems can not be done anaytically.

\section{ACKNOWLEDGMENTS}

Finacial support from the National Science Council under Grant No. NSC 92-2611-E-022-004 for the third author (I. L. Chen) is gratefully acknowledged.

\section{REFERENCES}

Atluri, S. N., and Zhu, T., 1998, “A New Meshless Local Petrov-Galerkin (MLPG) Approach in Computational Mechanics," Computational Mechanics, Vol. 22, No. 2, pp. 117-127.

Belystcho, T., Lu, Y., and Gu, L., 1994, "Element Free Galerkin Methods," International Journal for Numerical Methods in Engineering, Vol. 37, pp. 229-256.

Beskos, D. E., 1991, Boundary element analysis of plates and shells, Springer-Verlag, Berlin.

Chang, J. R., Yeih, W., and Chen, J. T., 1999, "Determination of Natural Frequencies and Natural Modes Using the Dual BEM in Conjunction with the Domain Partition Technique," Computational Mechanics, Vol. 24, No. 1, pp. 29-40.

Chen, C. S., Golberg, M. A., and Hon, Y. C., 1998, "The Method of Fundamental Solutions and Quasi-Monte-Carlo Method for Diffusion Equations," International Journal for Numerical Methods in Engineering, Vol. 43, No. 8, pp. 14211435.

Chen, I. L., Chen, J. T., Kuo, S. R., and Liang, M. T., 2001, "A New Method for True and Spurious 
Eigensolutions of Arbitrary Cavities Using the CHEEF Method," Journal of Acoustical Society of America, Vol. 109, No. 3, pp. 982-999.

Chen, J. T., 2000a, "Recent Development of Dual BEM in Acoustic Problems," Computer Methods in Applied Mechanics and Engineering, Vol. 188, No. 4, pp. 833-845.

Chen, J. T., Chang, M. H., Chen, K. H., and Lin, S. R., 2002, "The Boundary Collocation Method with Meshless Concept for Acoustic Eigenanalysis of Two-Dimensional Cavities Using Radial Basis Function," Journal of Sound and Vibration, Vol. 257, No. 4, pp. 667-711.

Chen, J. T., Chang, M. H., Chen, K. H., and Chen, I. L., 2002, "Boundary Collocation Method for Acoustic Eigenanalysis of Three-Dimensional Cavities Using Radial Basis Function," Сотриtational Mechanics, Vol. 29, Nos. 4-5, pp. 392408.

Chen, J. T., Chen, I. L., Chen, K. H., and Lee, Y. T., 2003, "Comment on Free Vibration Analysis of Arbitrarily Shaped Plates with Clamped Edges Using Wave-Type Function," Journal of Sound and Vibration, Vol. 262, pp. 370-378.

Chen, J. T., Chen, I. L., Chen, K. H., Lee, Y. T., and Yeh, Y. T., 2004, "A Meshless Method for Free Vibration Analysis of Circular and Rectangular Clamped Plates Using Radial Basis Function," Engineering Analysis with Boundary Elements, Vol. 28, pp. 535-545.

Chen, J. T., Huang, C. X., and Chen, K. H., 1999, "Determination of Spurious Eigenvalues and Multiplicities of True Eigenvalues Using the Real-Part Dual BEM," Computational Mechanics, Vol. 24, No. 1, pp. 41-51.

Chen, W., 2000b, "New RBF Collocation Schemes and Kernel RBF with Applications," Lecture Notes in Computational Science and Engineering, Vol. 26, pp. 73-84.

Chen, W., 2000c, "Symmetric Boundary Knot Method," Engineering Analysis with Boundary Elements, Vol. 26, No. 6, pp. 489-494.

Chen, W., and Tanaka, M., 2000a, "A Meshless, Exponential Convergence, Integration-Free, and Boundary-Only RBF Technique," Computers and Mathematics with Applications, Vol. 43, pp. 379391.

Chen, W., and Tanaka, M., 2000b, "Relationship Between Boundary Integral Equation and Radial Basis Function," Invited talk to the 52th Symposium of Japan Society for Computational Methods in Engineering (JASCOME) on BE\}, Tokyo.

Fairweather, G., and Karageorghis, A., 1998, "The Method of Fundamental Solutions for Elliptic Boundary Value Problems," Advances in Computational Mathematics, Vol. 9, Nos. 1-2, pp.
69-95.

Gingold, R. A., and Maraghan, J. J., 1977, "Smoothed Particle Hydrodynamics: Theory and Applications to Non-Spherical Stars," Monthly Notices of the Royal Astronomical Society, Vol. 181, No. 2, pp. 375-389.

Golberg, M. A., Chen, C. S., and Ganesh, M., 2000, "Particular Solutions of 3D HelmholtzType Equations Using Compactly Supported Radial Basis Functions," Engineering Analysis with Boundary Element, Vol. 24, pp. 539-547.

Hon, Y. C., and Chen, W., 2003, "Boundary Knot Method for 2D and 3D Helmholtz and Convection-Diffusion Problems under Complicated Geometry," International Journal for Numerical Methods in Engineering, Vol. 56, No. 13, pp. 1931-1948.

Hutchinson, J. R., 1988, "Vibration of Plates," Boundary Elements X, C. A., Brebbia, ed., Springer, Berlin, Germany, Vol. 4, pp. 415-430.

Kang, S. W., and Lee, J. M., 2000a, "Application of Free Vibration Analysis of Membranes Using the Non-Dimensional Dynamic Influence Function," Journal of Sound and Vibration, Vol. 234, No. 3, pp. 455-470.

Kang, S. W., and Lee, J. M., 2000b, "Eigenmode Analysis of Arbitrarily Shaped Two-Dimensional Cavities by the Method of Point Matching," Journal of Acoustical Society of America, Vol. 107, pp. 1153-1160.

Kang, S. W., and Lee, J. M., 2001, "Free Vibration Analysis of Arbitrary Shaped Plates with Clamped Edges Using Wave-Type Functions," Journal of Sound and Vibration, Vol. 242, No. 1, pp. 9-26.

Karageorghis, A., 2001, "The Method of Fundamental Solutions for the Calculation of the Eigenvalues of the Helmholtz Equation," Applied Mathematics Letter, Vol. 14, pp. 837-842.

Kitahara, M., Boundary Integral Equation Methods in Eigenvalue Problems of Elastodynamics and Thin Plates, Elsevier, Amsterdam, 1985.

Kupradze, V. D., 1964, "A Method for the Approximate Solution of Limiting Problems in Mathematical Physics," Computational Mathematics and Mathematical Physics, Vol. 4, No. 2, pp. 199-205.

Kuo, S. R., Chen, J. T., and Huang, C. X., 2000, “Analytical Study and Numerical Experiments for True and Spurious Eigenequations of a Circular Cavity Using the Real-Part Dual BEM," International Journal for Numerical Methods in Engineering, Vol. 48, No. 9, pp. 1401-1422.

Lee, Y. T., Chen, I. L., Chen, K. H., and Chen, J. T., 2002, "A New Meshless Method for Free Vibration Analysis of Plates Using Radial Basis Function," Proceedings of the 26th National Conference on Theoretical ans Applied Mechanics, 
Taiwan, R.O.C.

Leissa, W., 1969, "Vibration of Plates," NASA SP160.

Liu, W. K., Jun, S., and Zhang, Y. F., 1995, "Reproducing Kernel Particle Methods," International Journal for Numerical Methods in Engineering, Vol. 20, pp. 1081-1106.

Mukherjee, Y. X., and Mukherjee, S., 1997, "The Boundary Node Method for Potential Problems," International Journal for Numerical Methods in Engineering, Vol. 40, No. 5, pp. 797-815.

Poullikkas, A., Karageorghis, A., and Georgiou, G., 1998, "Method of Fundamental Solutions for
Harmonic and Bihramonic Boundary Value Problems," Computational Mechanics, Vol. 21, Nos. 4-5, pp. 416-423.

Sladek, V., Sladek, J., Atluri, S. N., and Van Keer, R., 2000, "Numerical Integration of Singularities in Meshless Implementation of Local Boundary Integral Equations," Computational Mechanics, Vol. 25, No. 4, pp. 394-403.

Manuscript Received: Nov. 11, 2003 Revision Received: Feb. 05, 2004 and Accepted: Feb. 18, 2004 